\title{
Programming complex regulation mechanisms through simple molecular assembly
}

Dominic Lauzon

University of Montreal https://orcid.org/0000-0002-7513-3233

Alexis Vallee-Belisle ( $\nabla$ a.vallee-belisle@umontreal.ca )

University of Montreal https://orcid.org/0000-0002-5009-7715

Article

Keywords: complex regulation mechanisms, simple molecular assembly, nanosystems, nanotechnologies

Posted Date: September 17th, 2020

DOl: https://doi.org/10.21203/rs.3.rs-75129/v1

License: (9) This work is licensed under a Creative Commons Attribution 4.0 International License. Read Full License 


\title{
Programming complex regulation mechanisms through simple molecular assembly
}

\author{
Authors: D. Lauzon ${ }^{1}$, A. Vallée-Bélisle ${ }^{1,2 *}$
}

\author{
Affiliations: \\ ${ }^{1}$ Laboratory of Biosensors \& Nanomachines, Département de Chimie, Université de Montréal, \\ Montréal, QC, H2V 0B3, Canada. \\ ${ }^{2}$ Département de Biochimie et Médecine Moléculaire, Université de Montréal, Montréal, QC, \\ H3T 1J4, Canada. \\ *Correspondence to: a.vallee-belisle@umontreal.ca
}

\begin{abstract}
What are the advantages and disadvantages of building nanosystems using one or multiple components? More than 55\% of all proteins found in living organisms are multimeric and likely exploit molecular assembly to create new functional entities. However, the specific contribution of molecular assembly to the creation of novel functions remains relatively unexplored at the thermodynamic, kinetic and molecular levels. Here, we use theory and a simple experimental model to determine the design rules for engineering efficient self-assembled, self-regulated nanosystems. Using these rules, we have rationally designed and implemented various regulation mechanisms (e.g., cooperative and anticooperative assembly, self-inhibition, molecular timer) into two model trimeric nanosystems including a complex artificial catalyst. These simple strategies based on molecular assembly have been extensively exploited by natural biosystems and are expected to play a crucial role in the development of future self-regulated nanotechnologies.
\end{abstract}


Finely regulated self-assembled molecular systems — aptly called nanomachines — are central to life and are increasingly important in nanotechnology $y^{1,2}$. In living organisms, molecular systems have evolved to respond precisely to specific variations in stimuli such as temperature, pressure, light, $\mathrm{pH}$, osmolarity, small molecules, or macromolecules ${ }^{3-5}$. These nanosystems typically self-assemble via the formation of multiple noncovalent interactions, either through intramolecular folding or through the intermolecular association of two or more molecular components ${ }^{6,7}$. The tetrameric protein hemoglobin, for example, contains four oxygencarrying components finely regulated by variation in $\mathrm{pH}$, carbon dioxide, and temperature and by the allosteric effector 2,3-bisphosphoglyceric acid (2,3-DPG) ${ }^{8}$. Inspired by such sophisticated nanosystems, chemists and engineers aspire to develop similar self-regulated systems for various nanotechnological applications, including biosensing, drug delivery and chemical computing ${ }^{9-11}$. Different mechanisms have been exploited by nature to create finely regulated molecular systems $^{12-14}$. Allostery, for example, regulates the activity of biological macromolecules through structural changes caused by the binding of an effector molecule at a location often distal to the active site ${ }^{15-17}$. An improved thermodynamic understanding of allosteric mechanisms has recently provided powerful strategies to optimize the performance of artificial nanosystems, such as their dynamic range, sensitivity, and cooperativity ${ }^{17-21}$. Another potentially important strategy to introduce regulation into molecular systems is by generating functional entities from the spontaneous assembly of multiple molecular components ${ }^{12,13}$. Although this strategy has been exploited extensively by nature, e.g., ribosome self-assembly ${ }^{22,23}$, its specific and detailed contribution to creating novel functionalities remains relatively unexplored at a thermodynamic, kinetic and molecular level ${ }^{24-26}$. To explore the potential of molecular assembly to create new functionalities, we designed and characterized a model artificial biomolecular system. Using 
theory and experiments, we demonstrate how simple self-assembled molecular systems can be readily programmed to produce complex regulation mechanisms by simply changing their number of components, their concentrations, and their thermodynamics and kinetics of assembly (Fig. 1A). We also apply this knowledge to engineer numerous regulation mechanisms into an artificially selected catalytic nanosystem.

A)
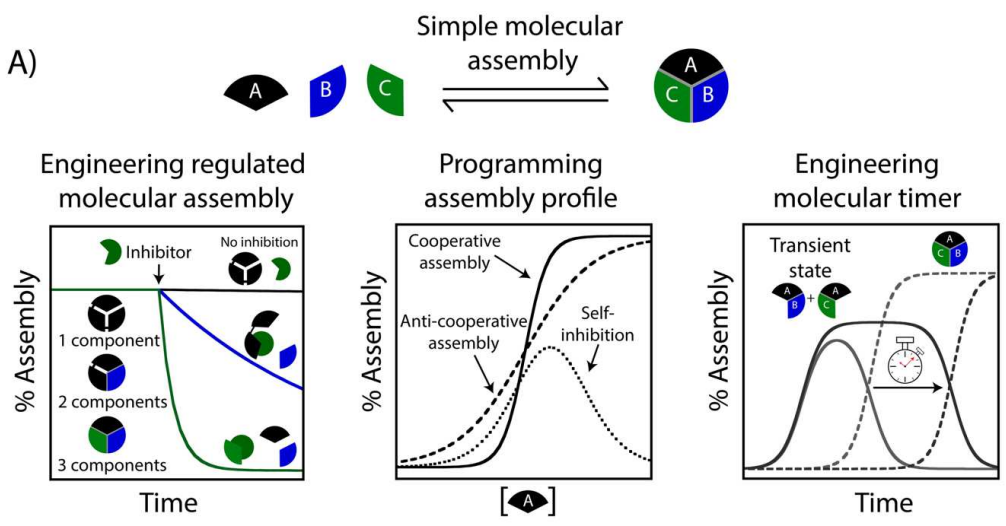

B)

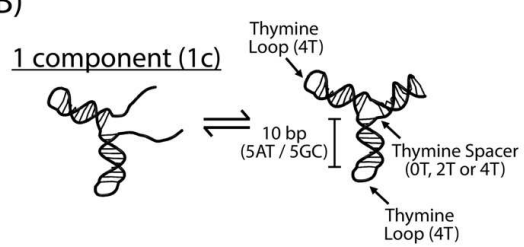

$\underline{2 \text { components }(2 \mathrm{c})}$

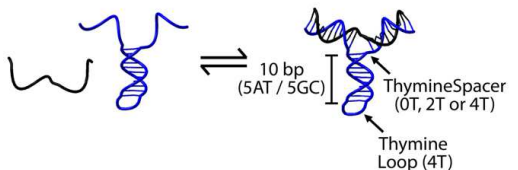

3 components (3c)

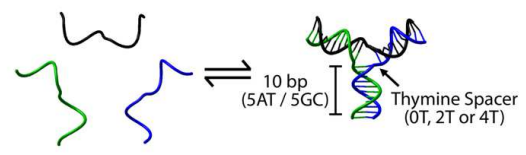

C)
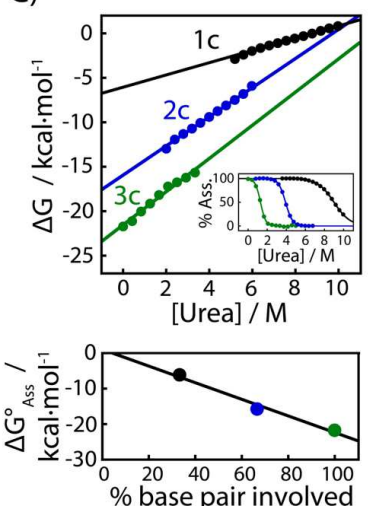

E)

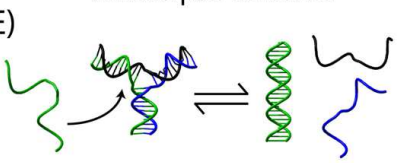

D)
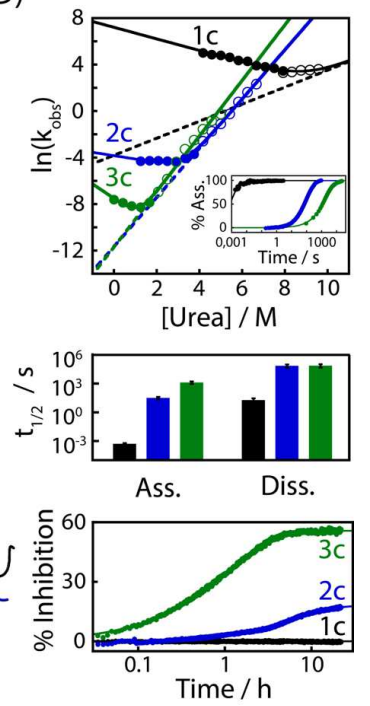

Figure. 1 | Designing molecular assemblies using one (black, 1c), two (blue, 2c) or three (green, 3c) molecular components. A) Molecular assemblies with multiple components enable the creation of various programmable assembly profiles (e.g., cooperative, anti-cooperative and self-inhibited assembly) with temporally controlled activation (e.g., molecular timer). B) A simple DNA-based self-assembled "3-way junction" nanosystem containing three 10 base pairs $(5 \mathrm{AT} / 5 \mathrm{GC})$ arms. All assemblies were monitored using fluorescently labeled DNA strands, and the data were normalized accordingly (see Methods). C) Top: Urea (and temperature, Fig. S5) denaturation curves reveal that the free energy of assembly $\left(\Delta \mathrm{G}^{\circ}\right.$ Ass $)$ increases with the number of components: $-5.9 \pm 0.6(1 \mathrm{c}),-15.8 \pm 0.2(2 \mathrm{c})$ and $-21.8 \pm 0.9 \mathrm{kcal} / \mathrm{mol}(3 \mathrm{c})$ (see inset and Fig. S2 
for raw data). Bottom: The $\Delta \mathrm{G}^{\circ}$ Ass and $m$-values (see Fig. S4) are proportional to the number of base pairs involved in the dissociation process (hairpins remain folded in the dissociated state). D) Top: Kinetics of assembly (closed symbols) and disassembly (open symbols) of all nanosystems under various urea concentrations (chevron plot) reveal a two-state mechanism (see Table S1). Bottom: The 1c system assembles and disassembles faster than the 2c and 3c systems (see also Fig. S6-S8 for raw data). E) Increasing the number of components increases the ability to inhibit the assembly process by using a complementary "inhibitor" strand.

\section{Results}

To establish the thermodynamic and kinetic rules of molecular assembly, we designed a simple self-assembled "3-way junction" nanosystem that can be readily built using 1,2 or 3 components (Fig. 1B). This widely occurring motif in natural and artificial ribozymes and DNAzymes $^{27}$ is also an important building block in DNA nanotechnology ${ }^{28,29}$. This 3-way junction contains three arms each separated by a short 2-thymine spacer (see Fig. S1 for linker design). Each arm is made from 10 base pairs $(5 \mathrm{AT} / 5 \mathrm{GC})$ and has a predicted folding free energy of $-10 \mathrm{kcal} / \mathrm{mol}\left(K_{D} \sim 100 \mathrm{nM}\right)$, which enables assembly within a range of concentrations ideally suited for fluorescence measurements ${ }^{30}$.

We first compared the stability of all 3-way junctions by assessing the difference in energy between the assembled and disassembled conformations, otherwise known as $\Delta \mathrm{G}$, using chemical denaturation procedures (see also SI for thermal denaturation procedure). Urea titration curves typically provide two important parameters: 1) an estimation of $\Delta \mathrm{G}$ in the absence of urea ( $\Delta \mathrm{G}^{\circ}$ Ass) and 2) the $m$-value, which correlates with the amount of surface area exposed to solvent upon disassembly (see Fig. 1C, Top) (1,32 $^{31}$ Surprisingly, we found that the one-component system (1c) is significantly less stable than the $2 \mathrm{c}$ and $3 \mathrm{c}$ systems $(-5.9 \pm 0.6 \mathrm{kcal} / \mathrm{mol}$ versus $-15.8 \pm 0.2$ and $-21.8 \pm 0.9 \mathrm{kcal} / \mathrm{mol}$, respectively). We also found that the $1 \mathrm{c}$ system exposes much less of its surface upon disassembly than the $2 \mathrm{c}$ and $3 \mathrm{c}$ systems $(m$-value $=0.67 \pm 0.06 \mathrm{kcal} / \mathrm{mol} \cdot \mathrm{M}$, $1.61 \pm 0.05 \mathrm{kcal} / \mathrm{mol} \cdot \mathrm{M}$, and $2.1 \pm 0.5 \mathrm{kcal} / \mathrm{mol} \cdot \mathrm{M}$, respectively) (Fig. S4). This is likely because 
the 1c system is already preorganized in its disassembled state. To verify this hypothesis, we characterized the isolated hairpins and found that they remain folded even above $10 \mathrm{M}$ urea (Fig. $\mathrm{S} 2)$. The $\Delta \mathrm{G}^{\circ}$ Ass and the $m$-values of the different systems are indeed proportional to the number of base pairs formed during the assembly/disassembly transition (Fig. 1B, Bottom and Fig. S4). Thermal denaturation of these nanosystems also revealed that the assembly of the 1c system showed both a reduction in enthalpy $(54 \pm 9 \%)$ and in entropy $(49 \pm 10 \%)$ compared to the $3 \mathrm{c}$ system, consistent with the idea that two of the three arms remain organized in the disassembled state of 1c (Fig. S5).

We then determined the kinetics of assembly of these nanosystems and found that their rate of assembly decreases drastically when the number of components was increased (Fig. 1D). For example, while $50 \%$ of the $1 \mathrm{c}$ system folds within $1 \mathrm{~ms}\left(t_{1 / 2}=0.5 \pm 0.1 \mathrm{~ms}\right)$, the $2 \mathrm{c}\left(t_{1 / 2}=32\right.$ $\pm 9 \mathrm{~s})$ and the $3 \mathrm{c}\left(t_{1 / 2}=1287 \pm 378 \mathrm{~s}\right)$ systems assemble five and seven orders of magnitude more slowly, respectively (Fig. 1D, inset). We also determined the half-life of all 3-way junctions and found that the $3 \mathrm{c}$ and $2 \mathrm{c}$ systems remained assembled up to four orders of magnitude longer than the $1 \mathrm{c}$ system (23 $\pm 9 \mathrm{~h}, 22 \pm 8 \mathrm{~h}$, and $21 \pm 11 \mathrm{~s}$, respectively) (Fig. 1D, Bottom). The $\Delta \mathrm{G}^{\circ}$ Ass and $m$-values estimated from the kinetics of assembly and disassembly are within the experimental 105 error of the values determined using equilibrium experiments, suggesting that each system assembles and disassembles via a two-state mechanism (see Fig. S4 and Table S1) ${ }^{33}$.

Overall, we found that despite its faster assembly rate, the unimolecular nanosystem is also more likely to form preorganized structures (e.g., hairpins) that do not contribute to the overall stability of the assembly. These preorganized structures always remain formed even 110 when the system is disassembled. In contrast, building nanosystems using many components reduces the level of preorganized structures, thus ensuring that the assembly mechanism 
maximizes the number of newly formed interactions. Preorganized structures in the disassembled state also impact the ability of the system to be regulated by simple allosteric mechanisms. For example, we found that the 1c system cannot be inhibited by a classic complementary DNA

115 "inhibitor" due to the low accessibility of its nucleotides locked in the hairpins. In contrast, the $2 \mathrm{c}$ and $3 \mathrm{c}$ systems are increasingly more sensitive to the presence of the inhibitor, thus displaying a better ability to develop more regulation mechanisms (Fig. 1E).

We then determined the impact of varying the number of components on the mechanism of assembly of these nanosystems. The assembly mechanism of unimolecular nanosystems, such as the 1c system, is generally hard to tune. These systems typically fold rapidly and become active as soon as they are synthesized ${ }^{34}$. Their activity, therefore, varies linearly with their concentration and cannot be regulated without the help of an external molecule, such as an allosteric effector (see Fig. S9) ${ }^{35,36}$. In contrast, the assembly of the dimeric $2 \mathrm{c}$ system can be regulated by tuning the concentration of one of its components (here called A). This results in a classic dose-response behavior (see Fig. 2A) with two programmable parameters: 1) the midpoint or $[\mathrm{A}]_{50 \%}$, i.e., the concentration at which $50 \%$ of the system is assembled, and 2 ) the cooperativity of the response or dynamic range (DR), i.e., the broadness of the transition, defined as the change in $[\mathrm{A}]$ required to provide a change in response from $10 \%$ to $90 \%(\mathrm{DR}=$ $\left.[\mathrm{A}]_{90 \%} /[\mathrm{A}]_{10 \%}\right)$. For example, when the $2 \mathrm{c}$ system assembles in the presence of a low concentration of one of its components (e.g., [B] $=1 \mathrm{nM}<K_{D}{ }^{A B}$, the dissociation constant of $\mathrm{AB}$, see Fig. $2 \mathrm{~A}$ lightest gray), its $[\mathrm{A}]_{50 \%}$ remains constant and equal to the dissociation constant of the system $\left([\mathrm{A}]_{50 \%}=K_{D}=5.6 \mathrm{nM}\right)$. In such cases, the dynamic range of the assembly remains approximately 81 -fold ( $69 \pm 12$-fold; see Fig. 2 A lightest gray). On the other hand, in a saturation regime, i.e., when the concentration of component B exceeds the $K_{D}$, the $[\mathrm{A}]_{50 \%}$ 
increases linearly with [B] $(0.5 \mathrm{x}[\mathrm{B}])$, indicating a 1:1 binding regime (see Fig. $2 \mathrm{C}$, left, blue curves) $)^{37}$. In such situations, the observed transition becomes more "cooperative", and the dynamic range is reduced by up to 9 -fold (Fig. 2C, right, blue curve).

The assembly of the trimeric $3 \mathrm{c}$ system displays even more programmability by enabling assembly over a wider range of concentrations. For instance, by varying the concentration of components B and C at levels lower or higher than $K_{D}{ }^{B C}(41 \pm 23 \mathrm{nM}$, see Fig. S10), one can tune their level of preorganization (i.e., $[\mathrm{BC}]$ ). For example, when using a concentration of B and $\mathrm{C}$ higher than $K_{D}{ }^{B C}$, the dimer preforms in a state similar to that of the $2 \mathrm{c}$ system, and consequently, the $3 \mathrm{c}$ system behaves similarly to the $2 \mathrm{c}$ system (Fig. 2A-B darker curves and Fig. 2C). In contrast, when using a concentration of $\mathrm{B}$ and $\mathrm{C}$ lower than $K_{D}{ }^{B C}$, the system is not 145 preorganized (i.e., B and $\mathrm{C}$ remain dissociated), and the assembly displays a much higher $[\mathrm{A}]_{50 \%}$ and dynamic range. For example, at $1 \mathrm{nM} B$ and $\mathrm{C}$ (Fig. 2B, lightest gray), the 3c systems display a $[\mathrm{A}]_{50 \%}$ of $92 \mathrm{nM}$ (vs $5.6 \mathrm{nM}$ for 2c) with a broad anticooperative dynamic range near 729-fold (vs 81-fold for 2c) (Fig. 2C). The assembly behaviors of the 2c and 3c systems are also well modeled by numerical simulations (see Fig. S9).

We can also conveniently tune the $[\mathrm{A}]_{50 \%}$ and the dynamic range of the $3 \mathrm{c}$ system by simply changing the affinity between the components and the ratio between their respective concentrations. For example, one can decrease (increase) the $[\mathrm{A}]_{50 \%}$ of the $3 \mathrm{c}$ system by simply decreasing (increasing) the temperature (Fig. 2D, left) or by increasing (decreasing) the number of Watson-Crick base pairs involved in the arms (Fig. 2D, middle). We also found that the anticooperative behavior observed at a low concentration of components $\mathrm{B}$ and $\mathrm{C}$ is highly dependent on the concentration ratio between these components $(\mathrm{R}=[\mathrm{C}] /[\mathrm{B}])$. As this ratio increases above 1, the anticooperativity rapidly disappears, and the system displays a typical 81- 
fold dynamic range (Fig. 2D, right). For example, a 2-fold increase in concentration of one component, from $\mathrm{R}=1$ to $\mathrm{R}=2$, decreases the dynamic range of assembly by up to 6-fold, from $\mathrm{DR}=383$ to $\mathrm{DR}=57$, while keeping the $[\mathrm{A}]_{50}$ relatively unchanged, from $18 \mathrm{nM}$ to $11 \mathrm{nM}$.

This provides a useful strategy to specifically program either the $[\mathrm{A}]_{50 \%}$ or the dynamic range independently. Overall, these results demonstrate that the assembly process of nanostructures made from many components can be programmed to provide much more diverse regulation profiles (e.g., from an anticooperative to a highly "cooperative" assembly profile).
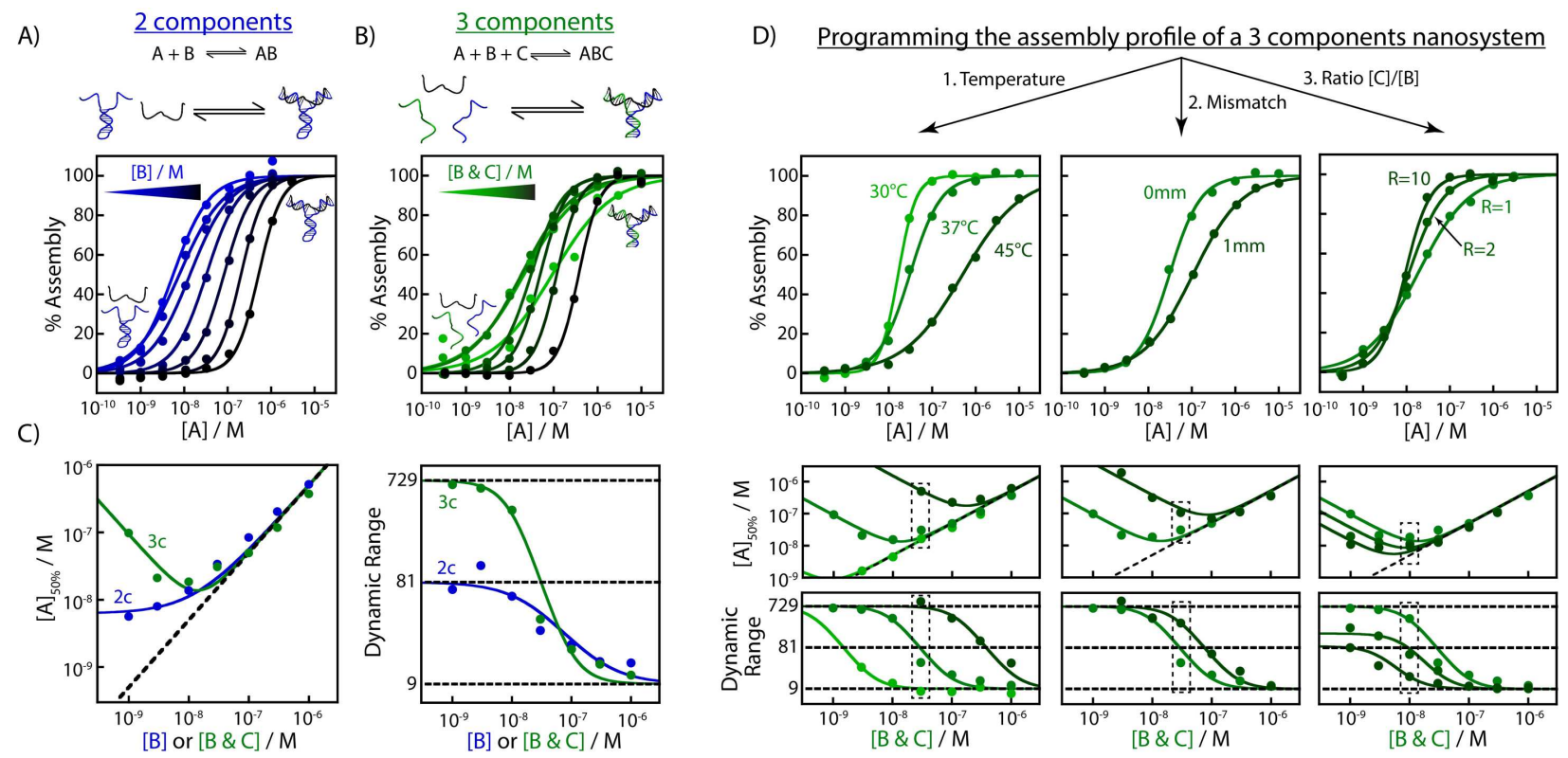

Figure. 2 | The assembly of nanosystems becomes more programmable (regulable) as the number of components increases. A) Programming the assembly ([A] $]_{50}$ and dynamic range) of $2 \mathrm{c}$ and B) $3 \mathrm{c}$ systems by increasing [A]. Assemblies are preformed at different fixed concentrations of strands B (2c) or strands B and C (3c). C) When the concentration of strand B is increased, the $[\mathrm{A}]_{50} \%$ of the $2 \mathrm{c}$ system increases from $5.6 \mathrm{nM}$ to $516 \mathrm{nM}$ (left), while its dynamic range decreases from 81- to 9-fold (right). When the concentrations of both strands B and $\mathrm{C}$ are increased, the $[\mathrm{A}]_{50 \%}$ of the $3 \mathrm{c}$ system displays a more complex relationship (changing from $92 \mathrm{nM}$ to $18 \mathrm{nM}$ to $372 \mathrm{nM}$-left), while its dynamic range decreases from 729- to 9-fold (right). D) The assembly profile of 3 components systems can be readily programmed by: 1) changing the temperature (left and Fig. S11); 2) introducing mismatch (middle and Fig. S12) or; 3 ) increasing the concentration ratio between the components B and C (right and Fig. S13). All dose-response curves are fitted using the Hill equation, while the $[\mathrm{A}]_{50 \%}$ and dynamic range are fitted using equations derived from the $2 \mathrm{c}$ and $3 \mathrm{c}$ models (see supplementary information). The 
180 experimental conditions were optimized to capture the whole assembly process over the experimentally allowed five orders of magnitude of [A].

Another way to program the assembly of a $3 \mathrm{c}$ system is by changing its propensity to form the final trimeric assembly $\left(\Delta \Delta \mathrm{G}_{\text {Tri-Dim }}\right.$ or $\left.\left|\Delta \mathrm{G}_{\text {Tri }}-\Delta \mathrm{G}_{\text {Dim }}\right|\right)$ by tuning the affinity of $\mathrm{A}$ towards the preorganized system BC (Fig. 3A). To explore and characterize this effect, we designed a set of three different 3-way junctions displaying similar $\Delta \mathrm{G}_{\text {Dim }}$ (same arms) but different $\Delta \mathrm{G}_{\text {Tri }}$ values. In an attempt to modulate only $\Delta \mathrm{G}_{\text {Tri }}$, we altered the stability of the junction by varying the linker length between the arms $(0,2,4 \text { thymines) (Fig. 1A })^{38,39}$. Using temperature denaturation curves, we found that all of our designs display similar dimeric affinities (approximately $-10.6 \pm 0.4 \mathrm{kcal} / \mathrm{mol}$, Fig. S1) but different trimeric affinities ranging from $-12.0 \pm 0.7 \mathrm{kcal} / \mathrm{mol}$ (0T spacer) to $-18.3 \pm 0.3 \mathrm{kcal} / \mathrm{mol}$ (2T spacer) (Fig. $3 \mathrm{~B}$ and Fig. S1D). As shown above, when using a concentration of B and $\mathrm{C}$ below $K_{D}{ }^{B C}$, we found that the most stable $3 \mathrm{c}$ system $\left(2 \mathrm{~T}\right.$ linker, $\left.\Delta \Delta \mathrm{G}_{\text {Tri-Dim }}=7.3 \pm 0.5 \mathrm{kcal} / \mathrm{mol}\right)$ assembles efficiently with a high level of "cooperativity" (Fig. $3 \mathrm{C}, \mathrm{DR}=20 \pm 5$ ). When $\Delta \Delta \mathrm{G}_{\text {Tri-Dim }}$ is significantly reduced

195 (e.g., $0 \mathrm{~T}$ linker, $\Delta \Delta \mathrm{G}_{\text {Tri-Dim }}=2.0 \pm 1.1 \mathrm{kcal} / \mathrm{mol}$ ), the assembly becomes self-inhibited ("none-allnone" mechanism, ${ }^{24}$ ) at high [A] through a mechanism that favors the formation of dimers over the trimer (Fig. 3E). For example, the percentage of assembled trimer increases from $10 \%$ to $70 \%$ when [A] is changed from $5 \mathrm{nM}$ to $200 \mathrm{nM}$ and goes back down to $10 \%$ when [A] is further increased to $10 \mu \mathrm{M}$ ( $\mathrm{DR}=2000$-fold; see also how to program this self-inhibited dynamic range in Fig. S14). This result highlights that if $\Delta \Delta \mathrm{G}_{\text {Tri-Dim }}$ is too small, an increase in [A] will not drive complex assembly further but rather inhibit it by favoring dimer formation (Fig. S14). Finally, the longer $4 \mathrm{~T}$ linker system, which produces a trimer of intermediate affinity $\left(\Delta \Delta \mathrm{G}_{\text {Tri-Dim }}=4.0 \pm\right.$ $0.8 \mathrm{kcal} / \mathrm{mol}$ ), displays less "cooperativity" of assembly than the more stable 2T system (Fig. 3C, 
$\mathrm{DR}=75 \pm 18$ ) without displaying the self-inhibition mechanism of the least stable 0T system. In contrast, similar mutations in the dimeric $2 \mathrm{c}$ system provided no diversity in the regulation mechanism (Fig. 3D).
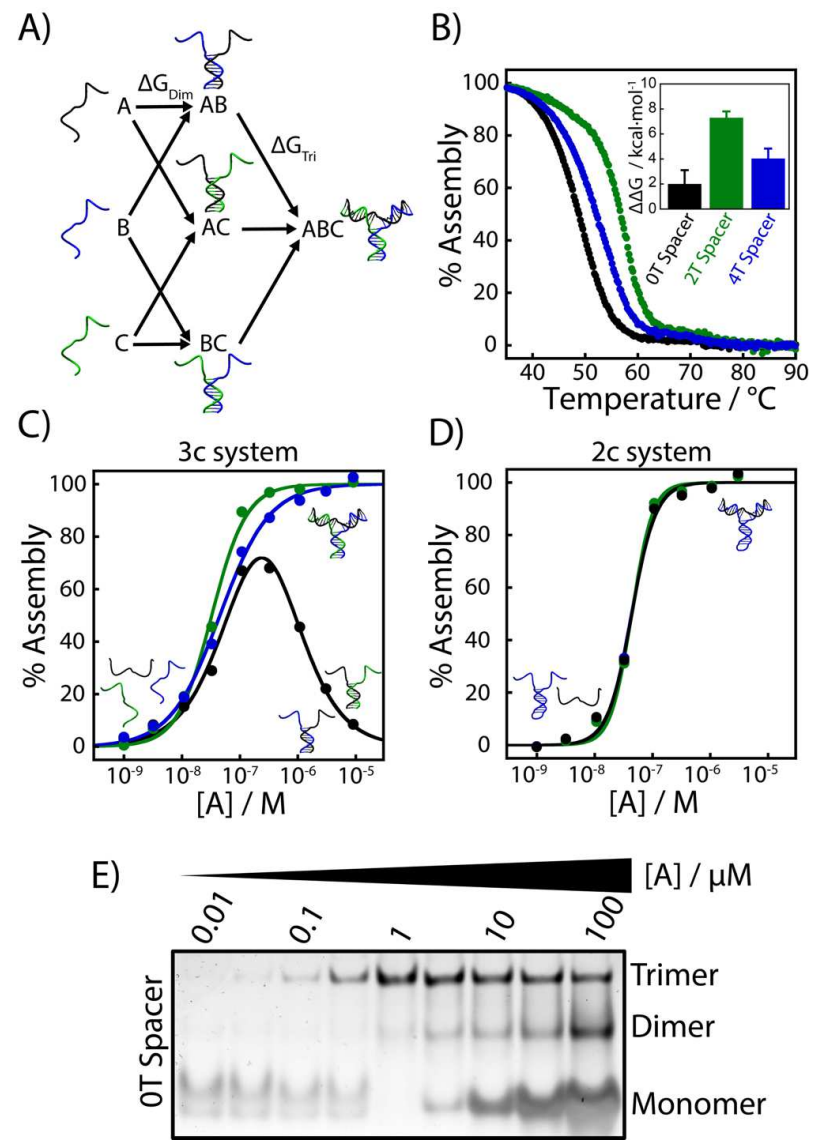

Figure. 3 | Programming trimer assembly by increasing the difference in energy between the dimer and trimer $\left(\Delta \Delta \mathbf{G}_{\text {Tri-Dim }}\right)$. A) Thermodynamic scheme of the assembly of the $3 \mathrm{c}$ 210 systems. B) Changing the thymine spacer length (0T black, 2T green and 4T blue, see Fig. 1A) creates trimers with different $\Delta \Delta \mathrm{G}_{\text {Tri-Dim }}$ values (see inset). C) Increasing $\Delta \Delta \mathrm{G}_{\text {Tri-Dim }}$ narrows the dynamic range of assembly (as seen with the more cooperative $2 \mathrm{~T}$ system), while low $\Delta \Delta \mathrm{G}_{\mathrm{Tr} i-D i m}$ $(0 \mathrm{~T})$ creates a self-inhibited trimeric system, which disassembles into two dimers ( $\mathrm{AB}$ and $\mathrm{AC}$ ) at higher [A]. D) Similar modifications on the 2c system provide no difference in assembly. E) 215 Polyacrylamide gel electrophoresis of the $0 \mathrm{~T}$ spacer trimeric assembly supports that the decrease in trimer occurs through sequestration into dimers (also see Fig. S15).

The regulation mechanisms discussed above all take place at equilibrium. However, molecular assemblies can also be under kinetic control ${ }^{40-42}$. In the case of the $3 \mathrm{c}$ system, for example, mixing an excess of component $\mathrm{A}(100$ to $600 \mathrm{nM})$ with smaller concentrations of $\mathrm{B}$ 
and $\mathrm{C}(10 \mathrm{nM})$ traps the latter into nonfunctional $\mathrm{AB}$ and $\mathrm{AC}$ dimers (Fig. 4A). Dissociation of these dimers is then required to enable trimer formation by the slow association of the formerly sequestered components B and C, thus resulting in biphasic kinetics (Fig. 4B and Fig. S16). We confirmed that the fastest phase, $k_{\mathrm{Dim}}$, represents the formation of dimers $\mathrm{AB}$ and $\mathrm{AC}$ given their linear dependency on the concentration of monomer A (Fig. 4C blue). In contrast, the slowest phase, $k_{\text {Trim, }}$, represents the formation of the trimer and is rate-limited by the formation of dimer $\mathrm{BC}$, thus explaining its insensitivity to the concentration of component A (Fig. 4C green and Fig. S17). This kinetically controlled mechanism of assembly provides interesting time-dependent formation/dissociation profiles for the dimer and the activation of the trimer. Experimental assessments (Fig. 4 D-E) and numerical simulations (Fig. S18-S20) also show that these profiles are easily programmable. For instance, by simply increasing $[\mathrm{A}]$, one can increase the rate of formation (activation) and the percentage of dimers $\mathrm{AB}$ and $\mathrm{AC}$ (Fig. 4D). In contrast, simulation and mutational analysis reveal that by increasing the concentrations of $[\mathrm{B}]$ and $[\mathrm{C}]$ and therefore their level of preorganization, one can increase the rate of assembly of the trimer (Fig. 4E and Fig. S17). Notably, increasing the rate of trimer formation also decreased the percentage of dimers transiently formed. These results demonstrate how a trimeric molecular assembly can be easily programmed to activate and deactivate within specific time ranges, thus acting as a molecular timer. Such time-dependent nanosystems are important in various biochemical processes, such as signal transduction, protein synthesis and the cell cycle and are likely to provide promising applications in future self-regulated nanotechnologies ${ }^{43-45}$. 


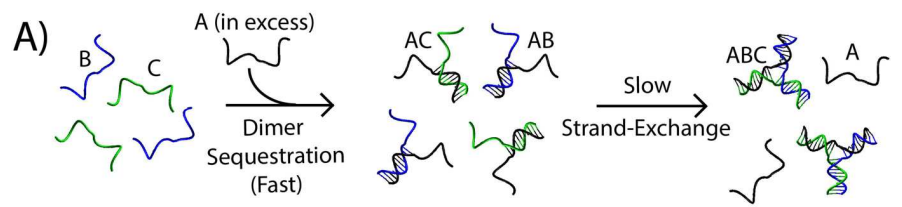

B)

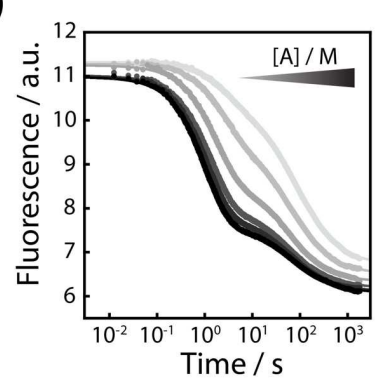

D)
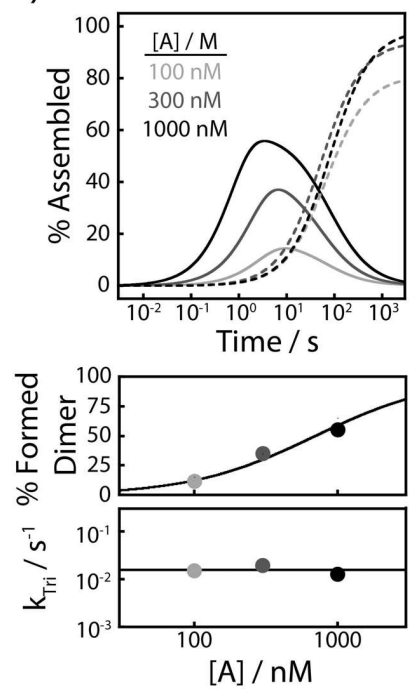

C)

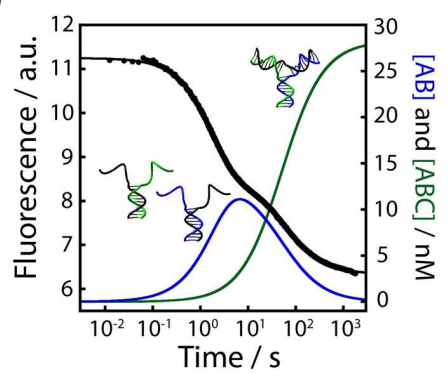

E)
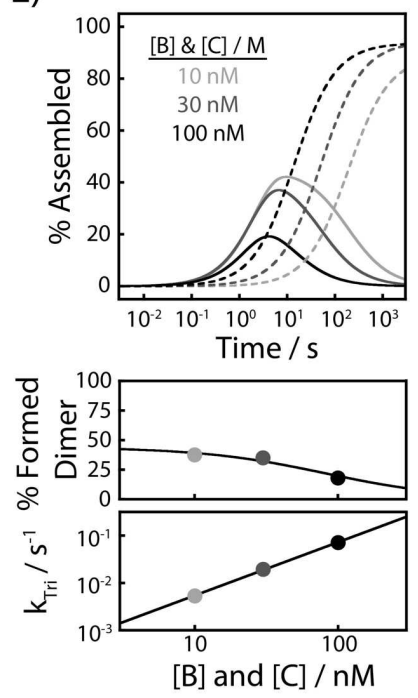

Figure. 4 | Programming time-dependent assembly. A-B) At low concentrations of monomers $\mathrm{B}$ and $\mathrm{C}$ (mainly unbound), the addition of an excess of monomer A produces slower biphasic kinetics due to the sequestration of strands $\mathrm{B}$ and $\mathrm{C}$ into $\mathrm{AB}$ and $\mathrm{AC}$ dimers. Trimer formation then proceeds through a slow strand-exchange mechanism limited by the association of the formerly sequestered components B and C (see Fig. S17 and S20). C) Kinetic trace of the dimeric (blue) and trimeric (green) assemblies derived from the raw kinetic data (black). D) By tuning the concentration of $\mathrm{A}$, one can program the rate of formation (activation) and the percentage of formed dimer without affecting the rate of trimeric assembly. E) By increasing the concentrations of $[\mathrm{B}]$ and $[\mathrm{C}]$ and therefore their level of preorganization, one can increase the rate of assembly of the trimer and decrease the percentage of transiently formed dimers. See Fig. S22 for raw data of panels D and E.

In this study, our 3-way junction served as a convenient synthetic toolkit to quantitatively test simple molecular assembly and the extent to which it can be harnessed to create novel regulation mechanisms. To test the generality and predictability of these findings, we used these 
rules to program the catalytic activity of $\mathrm{NaA} 43$, a sodium-specific RNA-cleaving DNAzyme previously used as a sensor to monitor the sodium concentration inside cells ${ }^{46}$. We first measured the apparent activity of NaA43 and found that it displays an apparent $K_{\mathrm{M}}$ of $16 \pm 1 \mathrm{nM}$, corresponding to half of the concentration of the DNAzyme used in our assay $(30 \mathrm{nM})$, and a dynamic range of $9 \pm 1$ (see Fig. 5A). We also estimated $K_{D}{ }^{D N A z y m e}$, the dissociation constant between the substrate and the DNAzyme, to be in the fM range (Fig. S23), which explains why this DNAzyme/substrate system operates in a saturation regime ([DNAzyme] $>K_{D}{ }^{D N A z y m e}$, see also Fig. 2A). To explore the effect of molecular assembly on the regulation of this DNAzyme, we engineered a dimeric DNAzyme by moving the loop far from the catalytic site, thus creating a three-component system. This modification still provides a functional DNAzyme despite a $49 \pm$ $4 \%$ reduction in catalytic activity (Fig. S24). At a high concentration of DNAzyme components $(1 \mu \mathrm{M})$, which ensures high preorganization, we found that $[\mathrm{A}]_{50 \%}$ is still approximately half the concentration of the DNAzyme $(729 \pm 29 \mathrm{nM})$ with a "cooperative" dynamic range (DR $=7.7 \pm$ 0.3). In contrast, as predicted by our model (Fig. 4A-B), using a low concentration of DNAzyme components (i.e., $30 \mathrm{nM}$ ) produces a kinetic trap. This kinetic trap can be used to program a substrate inhibition regulation mechanism (i.e., "none-all-none" regulation), where both DNAzyme components become sequestered into nonfunctional dimers at high substrate concentrations (see Fig. S25). 

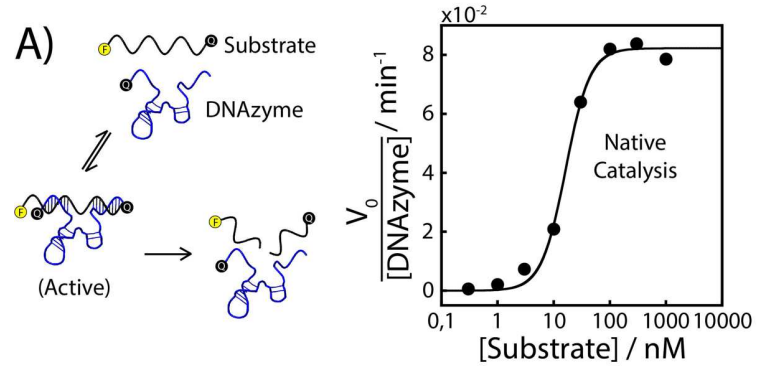

B)

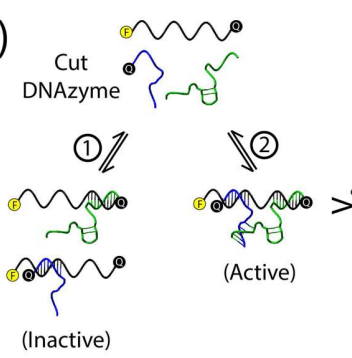

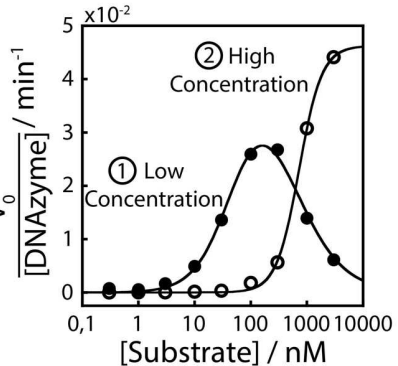

"Three-Component" DNAzyme System (Dimer Active)

C)

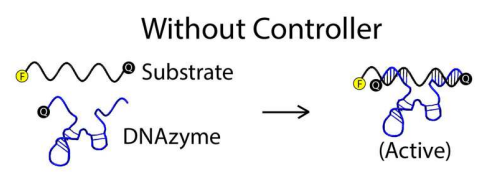

With Controller
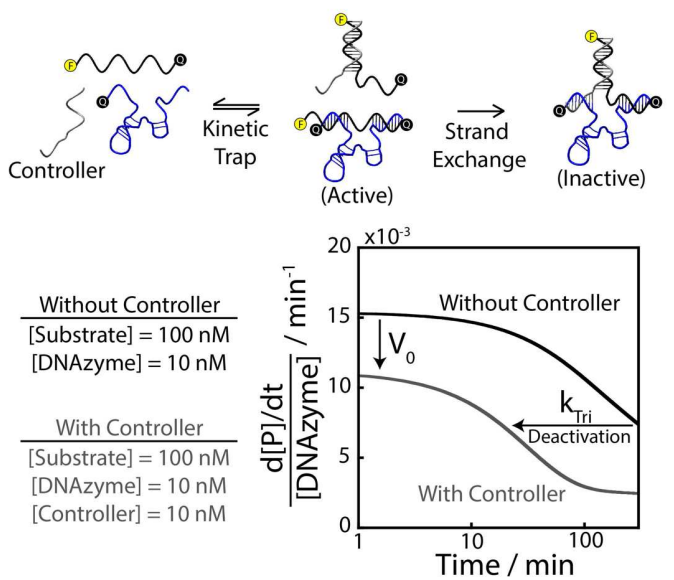

D)
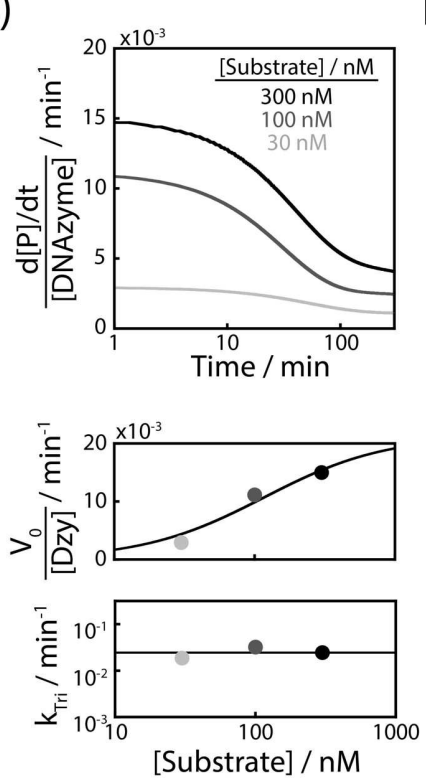

Figure 5 | Programming complex regulation mechanisms in a catalytic nanosystem through simple molecular assembly. A) The NaA43 cleaving DNAzyme ${ }^{46}$ displays an $[\mathrm{A}]_{50 \%}$ of $16 \pm 1$ $\mathrm{nM}$ at $30 \mathrm{nM}$ DNAzyme and a dynamic range of $9 \pm 1$ consistent with a saturation regime. B) When creating a trimeric assembly by cutting the DNAzyme, one can create a kinetic trap at a low concentration of DNAzyme $(30 \mathrm{nM})$ that leads to high dimer sequestration and lower activity. However, if the DNAzyme dimer is already preorganized $(1 \mu \mathrm{M})$, the system once again displays a saturation regime profile $\left([\mathrm{A}]_{50 \%}=729 \pm 29 \mathrm{nM}\right.$ and $\left.\mathrm{DR}=7.7 \pm 0.3\right)$. For raw data, see Fig. S26. C) We can also create a trimeric assembly by introducing a controller strand that can interact with both the native DNAzyme and the substrate. This controller strand can modulate the level of activity and the deactivation time through the formation of an inactive trimer (bottom). D) In the presence of $10 \mathrm{nM}$ controller and DNAzyme, increasing the concentration of substrate increases the formation of the active dimer and the catalytic rate (top and middle panel) without significantly affecting the trimeric deactivation rate $k_{\text {Tri }}$ (bottom panel). E) At $100 \mathrm{nM}$ substrate, increasing the concentration of controller and DNAzyme increases the rate of trimer formation and thus the rate of deactivation of the DNAzyme (bottom panel). Of note, the rates shown in panels C-E represent the derivatives of the fitted raw data (see Fig. S29). 
We then programmed the DNAzyme into a molecular timer to allow its activity to be finely regulated over time. To do this, we created a novel trimeric assembly by adding (instead of cutting, see Fig. 5B) an extra component to the existing system (Fig. 5C). We designed this extra “controller” component to hybridize to a variant of the DNAzyme (see Fig. S27 for design), forming either an inactive trimer or an active dimer (Fig. 5C top and Fig. S28). In contrast to the native DNAzyme, which deactivates only when running out of substrate, our molecular assembly strategy enables tuning of both the catalytic rate and the deactivation time of the DNAzyme (Fig. 5C bottom). As depicted, by further increasing the substrate concentration, we favor dimer sequestration and formation of the active DNAzyme, thus increasing the catalytic rate (Fig. 5D). This dimer deactivates at a controlled time $t_{1 / 2}=29 \pm 8$ min upon binding to the controller strand (forming the inactive trimer). In contrast, by increasing the concentration of DNAzyme and controller (Fig. 5E), we favor a faster assembly of the trimer, leading to faster deactivation of the DNAzyme without drastically changing the amount of dimer generated (i.e., with a similar catalytic rate). These results exemplify how simple molecular assembly strategies can be applied to introduce finely programmed regulation mechanisms into complex nanosystems such as a DNAzyme.

\section{Discussion}

Here, we have demonstrated the versatility of simple molecular assembly to achieve a wide range of regulatory mechanisms in two different model nanosystems. We have shown that despite assembling at a slower rate, nanosystems built using multiple components may also lead to assemblies that are significantly more stable. They do so by employing smaller components that contains less preorganized structures, thereby leading to more interactions being formed during the assembly process (Fig. 1). Smaller, less preorganized components also display more 
potential to form interactions with other molecular effectors, thus creating novel avenues for regulating their assembly (e.g., complementary DNA inhibitor, Fig. 1D). Another advantage of three-component systems over two-component systems is that they permit assembly using both a 320 "cooperative" and an anticooperative process (Fig. 2). We also showed that three-component systems can be tuned to exhibit self-inhibition mechanisms (Fig. 3) as well as time-dependent activation/deactivation mechanisms (Fig. 4). All these complex regulation profiles (at equilibrium or over time) are achievable using only a simple molecular assembly strategy and can be applied to systems of increasing complexity (e.g., catalytic nanosystem, Fig. 5). This

325 illustrates the simplicity of this approach compared to the more complex allosteric regulation mechanisms often employed by nature to produce similar regulation profiles ${ }^{19,47,48}$.

Engineering complex self-regulation mechanisms using simple molecular assembly provides a quantitative and programmable chemical strategy to develop and optimize nanosystems with applications ranging from biosensing ${ }^{49}$ to chemical computing ${ }^{50,51}$ and drug 330 delivery ${ }^{52,53}$. For example, current strategies to extend the dynamic range of sensors consist of combining two or multiple sensors with different affinities ${ }^{54-56}$. In contrast, here, we have illustrated how a three-component sensing system can be programmed to display either a narrow or an extended dynamic range. This ability to tune the dynamic range can also be useful to program and optimize the response of molecular logic gates. For example, a narrow dynamic range creates a more efficient all-or-none response, while a self-inhibited nanosystem (Fig. 3C) produces a "bandpass" filter (i.e., none-all-none response), a regulatory mechanism observed in many cellular functions ${ }^{57}$. A three-component system could also help maintain drug concentration within a specific therapeutic window. Nature, for example, employs various substrate inhibition strategies to maintain the level of crucial product metabolites despite large 
variations in substrate concentration ${ }^{58,59}$. One could envisage a simple three-component nanosystem to control and maintain the level of an active drug following its activation through a regulated catalytic system (Fig. 5B). Finally, a three-component system with programmed kinetic traps can enable time-specific activation/deactivation of various active biomolecules, leading to flexible and custom disease treatment strategies (Fig. 5C) $)^{60,61}$.

In addition to providing new strategies to develop complex self-regulated nanosystems, elucidating the thermodynamic and kinetic basis of molecular assembly will likely contribute to a better understanding of protein complex evolution. We have demonstrated how two common natural mechanisms to create novel protein assemblies, fission (i.e. cut in half) and fusion (i.e. controller strand $)^{62,63}$, have enabled us to engineer novel functionalities in a DNAzyme. Given 350 that more than $55 \%$ of all proteins in living organisms are multimeric ${ }^{26}$, it will be interesting to explore whether the functional gains of these proteins, e.g., complex regulation mechanisms, have emerged from the advantages derived from simple molecular assembly strategies ${ }^{64}$. It remains challenging to answer this question, however, given that multimeric proteins have evolved and diverged over billions of years ${ }^{26,65}$. In conclusion, simple molecular assembly 355 provides an efficient, programmable strategy to improve nanosystem functionality (e.g., by enabling optimized regulation mechanisms). We believe that the simplicity with which this strategy can be implemented in any nanosystem will greatly impact the development of future self-regulated nanotechnologies ${ }^{40,66}$. 


\section{Methods}

Chemicals. Anhydrous acetonitrile, activation reagents (0.25 M ETT in ACN), oxidizer (0.1 M I2 in H2O/Pyridine/THF), deblock solution (3\% TCA in DCM), Cap A mix (Ac2O/Pyridine/THF) and Cap B mix (16\% MeIm in THF) were purchased from ChemGenes Corporation, Wilmington. dA-CE phosphoramidite (n-bz), dC-CE phosphoramidite (n-bz), dGCE phosphoramidite (n-ibu), dT-CE phosphoramiditeand2'-TBDMS rA-CE phosphoramidite (nbz) were purchased from ChemGenes Corporation, Wilmington. Pre-pack $1000 \AA$ CPG synthesis columns of $\mathrm{dA}(\mathrm{Bz}), \mathrm{dC}(\mathrm{Bz}), \mathrm{dG}(\mathrm{DMF}), \mathrm{dT}$ and BHQ-1 were purchased from Biosearch Technologies, Novato. Fluorescein T amidite was purchased from Biosearch Technologies, Novato. MicroPure II columns for purification were purchased from Biosearch Technologies, Novato. Ammonium hydroxide solution (ACS, 28.0-30.0\%) and acetic acid (ACS, $\geq 99.7 \%$ ) were purchased from Sigma Aldrich. Triethylamine was purchased from Fisher Scientific. Trifluoroacetic acid (99\%) and triethylamine $3 \mathrm{HF}$ were purchased from Alfa Aesar. Ammonium bicarbonate and DMSO were purchased from BioShop. Acrylamide, bis-acrylamide, TEMED and ammonium persulfate were purchased from Bioshop. Milli-Q water was prepared in the lab when needed. All buffer solutions were prepared in the lab.

Oligonucleotides Synthesis. DNA/RNA synthesis is performed in the lab using standard phosphoramidite chemistry with the DNA/RNA synthesizer H-6 (K\&A Laborgeraete, Schaafheim). Cleavage from the solid support and deprotection of the bases are done overnight (16h) at $60^{\circ} \mathrm{C}$ in $28.0-30.0 \%$ aqueous ammonia. Purification of DNA strand is done by solidphase extraction using the P-8 purifier from K\&A Laborgeraete, Schaafheim. Purification protocol follows the K\&A Laborgeraete's method. For strand containing a ribonucleotide, a second deprotection step is required prior to purification. Solutions are dried and the deprotection 
of the 2'-TBDMS of the ribonucleotide is done for $1 \mathrm{~h} 30$ at $60^{\circ} \mathrm{C}$ in $250 \mu \mathrm{L}$ of TEA $3 \mathrm{HF}$ cocktail (1.5 $\mathrm{mL}$ DMSO, $750 \mu \mathrm{L}$ TEA and $1 \mathrm{~mL}$ TEA $3 \mathrm{HF}$ ). The mixture is cooled down to room temperature then quenched with $1 \mathrm{~mL}$ of $1.5 \mathrm{M}$ ammonium bicarbonate. Solutions are passed through a buffer exchange column (NAP ${ }^{\mathrm{TM}}-10$ column Sephadex ${ }^{\mathrm{TM}} \mathrm{G}-25$ DNA grade) prior to purification by HPLC (1260 infinity, Agilent Technologies) with a reversed-phase column (XBridge ${ }^{\circledR}$ Oligonucleotide BEH C18 OBD ${ }^{\mathrm{TM}}$ Prep column, $130 \AA, 2.5 \mu \mathrm{m}, 10$ x $50 \mathrm{~mm}$ ). Purification protocol follows Waters' recommendation. After purification, all DNA/RNA strand are dried with a Genevac ${ }^{\mathrm{TM}}$ miVac centrifugal concentrator, resuspended in Milli-Q water and quantified by UV-Vis (Cary 60, Agilent Technologies). Absorption coefficients ( $\varepsilon$ ) are estimated 395 from the DNA sequence using the IDT OligoAnalyzer tool.

\section{Sequence.}

$\underline{1 c \text { system }}$

Strand ABC with 2T spacer and 4T loop: 5'- GTA GTT CGA GTT CTT GAC GTA CTT TTG TAC GTC AAG TTG TAC ATC AGC TTT TGC TGA TGT ACT TCT CGA ACT AC $-\underline{F A M}-3^{\prime}$

$400 \quad \underline{2 c \text { system }}$

Strand A with 2T spacer: 5'- GCT GAT GTA CTT CTC GAA CTA C - $(\underline{F A M})-3^{\prime}$

Strand BC with 2T spacer: 5'- GTA GTT CGA GTT CTT GAC GTA CTT TTG TAC GTC AAG TTG TAC ATC AGC -(BHQ1)-3'

$\underline{3 \mathrm{c} \text { system }}$

405 Strand A 0T spacer: 5'- GCT GAT GTA CCT CGA ACT AC - $\underline{B H Q 1-3 '}$

Strand A 2T spacer: 5'- GCT GAT GTA CTT CTC GAA CTA C - $\underline{B H Q 1-3 '}$

Strand A 4T spacer: 5'- GCT GAT GTA CTT TTC TCG AAC TAC - $\underline{B H Q 1-3 '}$

Strand B 0T spacer: 5'- GTA GTT CGA GCT(FAM) TGA CGT AC -3' 
Strand B 2T spacer: 5'- GTA GTT CGA GT $\underline{\text { (FAM) }}$ CTT GAC GTA C -3'

Strand B 4T spacer: 5'- GTA GTT CGA GT $\underline{T(F A M)}$ TTC TTG ACG TAC -3'

Strand C 0T spacer: 5'- GTA CGT CAA GGT ACA TCA GC -3'

Strand C 2T spacer: 5'- GTA CGT CAA GTT GTA CAT CAG C -3'

Strand C 4T spacer: 5'- GTA CGT CAA GTT TTG TAC ATC AGC -3'

Strand A 2T spacer mismatch 1: 5'- $\underline{\mathbf{C} C T}$ GAT GTA CTT CTC GAA CTA $\underline{\mathbf{G}}-\underline{B H Q 1}-3^{\prime}$

415 Strand A 2T spacer mismatch 2: 5'- GCT GA $\underline{\mathbf{A}}$ GTA CTT CTC GIA CTA C - $\underline{B H Q 1-3 '}$

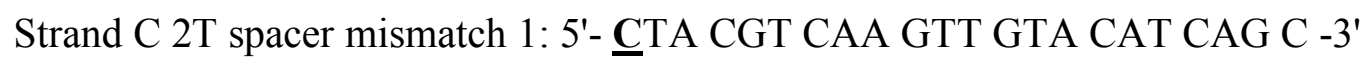

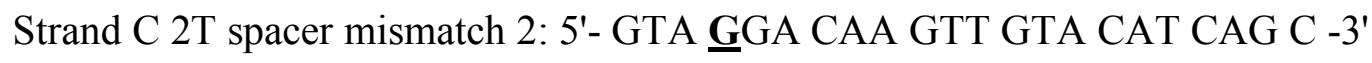

DNAzyne

Native DNAzyme (NaA43): 5'- GCG GCG GTA CCA GGT CAA AGG TGG GTG AGG GGA

CGC CAA GAG TCC CCG CGG TTA CAT AGA G - $\underline{B H Q 1-3 '}$

Cut DNAzyme 1: 5'- GCG GCG GTA CCA GGT CAA AGG TGG GTG AGG GGA CGC C -3'

Cut DNAzyme 2: 5'- AAG AGT CCC CGC GGT TAC ATA GAG - $\underline{B H Q 1-3 '}$

Modified DNAzyme (NaA43): 5'- GCㅡ GCG GTA CCA GGT CAA AGG TGG GTG AGG

GGA CGC CAA GAG TCC CCG CGG TTA CAT AG $\underline{\text { CGT C }}$ - $\underline{B H Q 1-3 '}$

Substrate: 5'-T(FAM)- CTG CTC TAT GTA TrAG GAA GTA CCG CCG CAT T - $\underline{\text { BHQ1 }}-3^{\prime}$

Controller: 5'- GAC GAC TAT GTA TGT TAC ATA GAG CAG -3'

In bold and underlined are the modifications relative to the native strand. FAM corresponds to a fluorescein, $T(F A M)$ corresponds to a thymidine conjugated with a fluorescein, $B H Q 1$ corresponds to a black hole quencher 1 and $r A$ corresponds to an adenosine. 
Urea Titration curves. Urea titration curves were performed following a method developed by our lab. ${ }^{31}$ We started with a $900 \mu \mathrm{L}$ solution of the DNA-based system of interest in $10 \mathrm{M}$ urea buffered solution $\left(10 \mathrm{mM} \mathrm{NaH}_{2} \mathrm{PO}_{4}, 40 \mathrm{mM} \mathrm{NaCl}, \mathrm{pH}=7.00\right)$. We then sequentially diluted this solution with a buffered solution containing the same concentration of DNA-based system but with no urea. Each sample was equilibrated for 2 minutes before recording their fluorescence (Cary Eclipse, Agilent Technologies). Unimolecular system titration ( $\left.\mathrm{A}_{\text {Diss }} \rightleftharpoons \mathrm{A}_{\mathrm{Ass}}\right)$ was performed at a concentration of $10 \mathrm{nM}$ of fluorescent DNA strand and fitted using Eq. 1 . Bimolecular system titration $(\mathrm{A}+\mathrm{B} \rightleftharpoons \mathrm{AB})$ was performed at a concentration of $10 \mathrm{nM}$ of fluorescent DNA strand and $100 \mathrm{nM}$ of the quencher DNA strand (10-fold excess) and fitted using Eq. 2. Trimolecular system titration $(\mathrm{A}+\mathrm{B}+\mathrm{C} \rightleftharpoons \mathrm{ABC})$ was performed at a concentration of $10 \mathrm{nM}$ of fluorescent DNA strand and $100 \mathrm{nM}$ of the quencher and unlabelled DNA strands (10-fold excess) and fitted using Eq. 3. In each equation, $F$ is the fluorescence signal, $F^{\circ}{ }_{A s s}$ and $F^{\circ}{ }_{\text {Diss }}$ are the intrinsic fluorescence of the assembled and disassembled states, $\sigma_{A s s}$ and $\sigma_{\text {Diss }}$ are the urea dependency of the fluorescence signal of the assembled and disassembled states, $[U]$ is 445 the concentration of urea, $\Delta G^{\circ}{ }_{A s s}$ is the Gibbs free energy of assembly in absence of urea, $\left[D_{Q}\right]$ and $\left[D_{\mathrm{Un}}\right]$ are the concentration of the quencher and unlabelled DNA strands (which are in 10time excess), $T$ is the temperature and $R$ is the gas constant.

$$
\begin{gathered}
F=\frac{\left(F_{A S S}^{\circ}+\sigma_{A S S}[U]\right)+\left(F_{D i s s}^{\circ}+\sigma_{D i s s}[U]\right) \cdot e^{-\frac{\left(\Delta G_{A S S}^{\circ}-m[U]\right)}{R T}}}{\left(1+e^{-\frac{\Delta G^{\circ} A s s}{R T}-m[U]}\right)} \\
F=\frac{\left[D_{Q}\right] \cdot\left(F_{A s S}^{\circ}+\sigma_{A s S}[U]\right)+\left(F^{\circ}{ }_{D i s s}+\sigma_{D i s s}[U]\right) \cdot e^{-\frac{\left(\Delta G_{A s s}^{\circ}-m[U]\right)}{R T}}}{\left(\left[D_{Q}\right]+e^{-\frac{\Delta G^{\circ} A s s^{-m[U]}}{R T}}\right)}
\end{gathered}
$$




$$
F=\frac{\left[D_{Q}\right] \cdot\left[D_{U n}\right] \cdot\left(F_{A s s}^{\circ}+\sigma_{A s S}[U]\right)+\left(F_{D i s S}^{\circ}+\sigma_{D i s s}[U]\right) \cdot e^{-\frac{\left(\Delta G^{\circ} A s s^{-m}[U]\right)}{R T}}}{\left(\left[D_{Q}\right] \cdot\left[D_{U n}\right]+e^{-\frac{\Delta G^{\circ} A s s^{-m[U]}}{R T}}\right)}
$$

Temperature melting curves. Thermodynamic constants measured using temperature melting curves were performed based on the work of Owczarzy et al.. ${ }^{67}$ The melting curves of all complexes are measured at different concentrations using a fluorimeter (Cary Eclipse, Agilent Technologies). All samples are heated to $90^{\circ} \mathrm{C}$ at $10^{\circ} \mathrm{C} / \mathrm{min}$ with a 2 minutes hold then cool down to $20^{\circ} \mathrm{C}$ at $10^{\circ} \mathrm{C} / \mathrm{min}$ with a 10 minutes hold prior the recording of the melting curve from $20^{\circ} \mathrm{C}$ to $90^{\circ} \mathrm{C}$ at $0.8^{\circ} \mathrm{C} / \mathrm{min}$. The $T_{m}$ is extracted from the derivative of the melting curve $(\mathrm{dF} / \mathrm{dT})$, which displays a "bell shape" curve. A Gaussian distribution fitting is used to evaluate the maximum of this $\mathrm{dF} / \mathrm{dT}$ plot. The thermodynamic parameters $\Delta \mathrm{H}$ and $\Delta \mathrm{S}$ are measures by linear regression (Eq. 4) and enables the calculation of $\Delta \mathrm{G}$. For all experiments, the concentration of the monomer with a fluorophore (called A) was kept constant at $1 \mu \mathrm{M}$, for $\Delta \mathrm{G}_{\text {Dim }}$ measurement, and $0.1 \mu \mathrm{M}$, for $\Delta \mathrm{G}_{T r i}$ measurement, while the concentration of the other monomer containing the quencher (called B) was varied between 5 and $200 \mu \mathrm{M}$ (for $\Delta \mathrm{G}_{\text {Dim }}$ measurement) and between 0.5 and $20 \mu \mathrm{M}$ (for $\Delta \mathrm{G}_{T r i}$ measurement). In the equation, $T m$ is the melting temperature, $\Delta H$ is the change in enthalpy, $\Delta S$ is the change in entropy, $[A]_{T}$ is the concentration of strand A and $[B]_{T}$ is the concentration of strand $\mathrm{B}$.

$$
\frac{1}{T_{m}}=\frac{R}{\Delta H} \ln \left([B]_{T}-\frac{[A]_{T}}{2}\right)+\frac{\Delta S}{\Delta H}
$$

Binding Curve. Appropriate dilutions are made such as the volume of the probe solution (strand containing the fluorophore) is always $900 \mu \mathrm{L}$. The probe solution contains either one strand (for the $2 \mathrm{c}$ system, called $B$ ) or two strands (for the $3 \mathrm{c}$ system, called $B$ and $C$ ). To this solution, a small volume of titrant (strand with a quencher, called $A$ ) is added and the fluorescence is 
recorded after 20 min of equilibration with a fluorimeter (Cary Eclipse, Agilent Technologies). The total volume of titrant added to the probe solution is kept below 5\% to avoid high dilution of the probe. We can then assume that the concentration of the probe remains approximately constant over the titration. The titration curve is then fitted using the Hill equation (Eq. 5) where $K_{o b s}$ is the observed dissociation constant (or the concentration at which $50 \%$ of the probe is bound, also called $\left.[\mathrm{A}]_{50 \%}\right), n_{H}$ is the Hill factor (an indicator of the cooperativity enabling the calculation of the dynamic range $\left.{ }^{20}\right), F$ is the fluorescence signal, $B s l$ is the baseline fluorescence and Amp is the amplitude (or change in fluorescence upon binding of the strand $A$ ). The dependency of $K_{o b s}$ and the dynamic range $(D R)$ over the concentration of probe is describe in detail in the supporting information.

$$
F=B s l+A m p \frac{[A]^{n_{H}}}{K_{o b s}{ }^{n_{H}}+[A]^{n_{H}}}
$$

Kinetic experiments. For fast kinetic ( $<30 \mathrm{~min})$, appropriate dilutions are made, and solutions of $A$ and $B$ are rapidly mixed using a stopped-flow instrument coupled with a fluorimeter (SX20, Applied Photophysics). Slower kinetics ( $>30 \mathrm{~min}$ ) are recorded using a standard fluorimeter (Cary Eclipse, Agilent Technologies). A small volume of solution $A$ is added with a pipette inside a cuvette containing solution $B$ and manually mixed using the same pipette. Oil is deposited on top of the solution to avoid water evaporation over the long kinetic measurement. DNA hybridization follows a second-order kinetic: $A+B \rightleftharpoons A B$ where $A$ and $B$ are singlestranded DNA and $A B$ is the duplex formed from these two strands ${ }^{68}$. Pseudo-first order kinetics are achieved by using at least a 10 -fold excess of strand A. The kinetic traces are then fitted using an exponential equation (Eq. 6) where $k_{o b s}$ is the first-order observed rate constant. The relationship of $k_{o b s}$ with the concentration of $\mathrm{A}$ is linear enabling the measurement of the association rate constant $\left(k_{\text {ass }}\right)$ and the dissociation rate constant $\left(k_{\text {diss }}\right)$. Second-order kinetics are 
achieved when using equimolar concentrations of $\mathrm{A}$ and $\mathrm{B}\left([A]_{O}=[B]_{o}\right)$. This system acts like a dimerization reaction (i.g. $2 \mathrm{~A} \rightleftharpoons \mathrm{A}_{2}$ ) and can thus be fitted using Eq. 7 where $k_{o b s}$ is now the second-order observed rate constant.

$$
\begin{gathered}
F=B s l+A m p \cdot e^{-k_{o b s} t} \\
F=B s l+A m p \cdot \frac{1}{1+2[A]_{o} k_{o b s} t}
\end{gathered}
$$

The trimeric association follows this reaction: $A+B+C \rightleftharpoons A B C$ where $A, B$, and $C$ are singlestranded DNA and $A B C$ is the three-way junction formed from these strands. When measuring the assembly, we use at least a 10 -fold excess of molecule $A$ in buffer that is rapidly mixed using a stopped-flow instrument coupled with a fluorimeter (SX20, Applied Photophysics) with a solution of $B$ and $C$ in water (to avoid pre-association of $B$ and $C$ ). Data are fitted using a combination of a pseudo-first-order kinetic and a second-order kinetic (Eq. 8).

$$
F=B s l+A m p_{1} \cdot e^{-k_{o b s, 2} t}+A m p_{2} \cdot \frac{1}{1+2[A]_{o} k_{o b s, 1} t}
$$

In all equation, $t$ is the time, $F$ is the fluorescence signal, $B s l$ is the baseline fluorescence, $A m p$ is the amplitude (or change in fluorescence upon binding) and $[A]_{O}$ is the concentration of strand A.

\section{Native PAGE experiments.}

Appropriate dilution of unlabeled DNA solutions were made such as the concentration of strands $\mathrm{B}$ and $\mathrm{C}$ is kept at $1 \mu \mathrm{M}$ and the concentration of strand $\mathrm{A}$ is changed from $30 \mathrm{nM}$ to $100 \mu \mathrm{M}$. Solutions are then mixed in a 5:1 ratio with the $6 x$ loading buffer $(2.5 \mathrm{mg} / \mathrm{mL}$ bromothymol blue, $2.5 \mathrm{mg} / \mathrm{mL}$ xylene cyanol $\mathrm{FF}$ and $30 \%$ glycerol in water). A $15 \%$ polyacrylamide gel is handcast following Bio-Rad protocol and incubated in the running buffer $(0.5 \mathrm{x}$ TBE buffer containing 5 $\mathrm{mM}$ of $\mathrm{MgCl}_{2}$ ) for $1 \mathrm{~h} .10 \mu \mathrm{L}$ of samples are run for $90 \mathrm{~min}$ at $120 \mathrm{~V}$ using the Mini-PROTEAN 
Tetra cell electrophoresis unit (Bio-Rad) and the Bio-Rad PowerPac Basic power supply. Gels are stained with a $0.5 \mathrm{x}$ solution of GelRed ${ }^{\mathrm{TM}}$ (Biotium) for 10 minutes and analyze on an imaging system (ChemiDoc ${ }^{\mathrm{TM}}$ XRS+, Bio-Rad). The integration of band intensity is then performed to evaluate the amount of assembled DNA-based systems.

\section{References}

515 1. Grzybowski, B. A., Huck, W. T. S. The nanotechnology of life-inspired systems. Nature Nanotechnology 11, 585 (2016).

2. Murphy, K. G., Bloom, S. R. Gut hormones and the regulation of energy homeostasis. Nature 444, 854-859 (2006).

3. Cameron, D. E., Bashor, C. J., Collins, J. J. A brief history of synthetic biology. Nature Reviews Microbiology 12, 381 (2014).

4. Benner, S. A., Sismour, A. M. Synthetic biology. Nature Reviews Genetics 6, 533 (2005).

5. Camilli, A., Bassler, B. L. Bacterial Small-Molecule Signaling Pathways. Science 311, 1113 (2006).

6. Marsh, J. A., Teichmann, S. A. Structure, Dynamics, Assembly, and Evolution of Protein Complexes. Annual Review of Biochemistry 84, 551-575 (2015).

7. Dill, K. A., MacCallum, J. L. The Protein-Folding Problem, 50 Years On. Science 338, 1042 (2012).

8. Benesch, R. E., Benesch, R. The Mechanism of Interaction of Red Cell Organic Phosphates with Hemoglobin. In: Advances in Protein Chemistry (eds Anfinsen, CB, Edsall JT, Richards FM). Academic Press (1974).

9. Erbas-Cakmak, S., et al. Molecular logic gates: the past, present and future. Chemical Society Reviews 47, 2228-2248 (2018).

10. Galés, C., et al. Real-time monitoring of receptor and G-protein interactions in living cells. Nature Methods 2, 177-184 (2005).

11. Pakulska, M. M., Miersch, S., Shoichet, M. S. Designer protein delivery: From natural to engineered affinity-controlled release systems. Science 351, aac4750 (2016).

12. Williamson, J. R. Cooperativity in macromolecular assembly. Nature Chemical Biology 4, 458 (2008).

13. Whitty, A. Cooperativity and biological complexity. Nature Chemical Biology 4, 435 (2008).

14. Wodak, S. J., et al. Allostery in Its Many Disguises: From Theory to Applications. Structure 27, 566-578 (2019).

15. Motlagh, H. N., Wrabl, J. O., Li, J., Hilser, V. J. The ensemble nature of allostery. Nature 508, 331-339 (2014).

$545 \quad$ 16. Vinkenborg, J. L., Karnowski, N., Famulok, M. Aptamers for allosteric regulation. Nature Chemical Biology 7, 519 (2011). 
17. Ricci, F., Vallée-Bélisle, A., Plaxco, K. W. High-Precision, In Vitro Validation of the Sequestration Mechanism for Generating Ultrasensitive Dose-Response Curves in Regulatory Networks. PLOS Computational Biology 7, e1002171 (2011).

18. Porchetta, A., Vallée-Bélisle, A., Plaxco, K. W., Ricci, F. Using Distal-Site Mutations and Allosteric Inhibition To Tune, Extend, and Narrow the Useful Dynamic Range of Aptamer-Based Sensors. Journal of the American Chemical Society 134, 20601-20604 (2012).

19. Ricci, F., Vallée-Bélisle, A., Porchetta, A., Plaxco, K. W. Rational Design of Allosteric Inhibitors and Activators Using the Population-Shift Model: In Vitro Validation and Application to an Artificial Biosensor. Journal of the American Chemical Society 134, 15177-15180 (2012).

20. Simon, A. J., Vallée-Bélisle, A., Ricci, F., Plaxco, K. W. Intrinsic disorder as a generalizable strategy for the rational design of highly responsive, allosterically cooperative receptors. Proceedings of the National Academy of Sciences 111, 1504815053 (2014).

21. Simon, A. J., Vallée-Bélisle, A., Ricci, F., Watkins, H. M., Plaxco, K. W. Using the Population-Shift Mechanism to Rationally Introduce "Hill-type" Cooperativity into a Normally Non-Cooperative Receptor. Angewandte Chemie International Edition 53, 9471-9475 (2014).

22. Karbstein, K. Mitochondria teach ribosome assembly. Science 365, 1077 (2019).

23. Woodson, S. A. RNA folding and ribosome assembly. Current Opinion in Chemical Biology 12, 667-673 (2008).

24. Ercolani, G., Schiaffino, L. Allosteric, Chelate, and Interannular Cooperativity: A Mise au Point. Angewandte Chemie International Edition 50, 1762-1768 (2011).

25. Garcia-Seisdedos, H., Empereur-Mot, C., Elad, N., Levy, E. D. Proteins evolve on the edge of supramolecular self-assembly. Nature 548, 244 (2017).

26. Lynch, M. The evolution of multimeric protein assemblages. Mol Biol Evol 29, 13531366 (2012).

27. Silverman, S. K. Catalytic DNA: Scope, Applications, and Biochemistry of Deoxyribozymes. Trends in Biochemical Sciences 41, 595-609 (2016).

28. Sadowski, J. P., Calvert, C. R., Zhang, D. Y., Pierce, N. A., Yin, P. Developmental SelfAssembly of a DNA Tetrahedron. ACS Nano 8, 3251-3259 (2014).

29. Hao, C., Li, X., Tian, C., Jiang, W., Wang, G., Mao, C. Construction of RNA nanocages by re-engineering the packaging RNA of Phi29 bacteriophage. Nature Communications 5, 3890 (2014).

30. Zuker, M. Mfold web server for nucleic acid folding and hybridization prediction. Nucleic Acids Research 31, 3406-3415 (2003).

31. Idili, A., Ricci, F., Vallée-Bélisle, A. Determining the folding and binding free energy of DNA-based nanodevices and nanoswitches using urea titration curves. Nucleic acids research 45, 7571-7580 (2017).

32. Lawrence, C., Vallée-Bélisle, A., Pfeil, S. H., de Mornay, D., Lipman, E. A., Plaxco, K. W. A comparison of the folding kinetics of a small, artificially selected DNA aptamer with those of equivalently simple naturally occurring proteins. Protein Sci 23, 56-66 (2014).

33. Jackson, S. E., Fersht, A. R. Folding of chymotrypsin inhibitor 2. 1. Evidence for a twostate transition. Biochemistry 30, 10428-10435 (1991). 
34. Plaxco, K. W., Simons, K. T., Ruczinski, I., Baker, D. Topology, Stability, Sequence, and Length: Defining the Determinants of Two-State Protein Folding Kinetics. Biochemistry 39, 11177-11183 (2000).

35. Wells, J. A., McClendon, C. L. Reaching for high-hanging fruit in drug discovery at protein-protein interfaces. Nature 450, 1001-1009 (2007).

36. Tan, J., et al. Regulation of Protein Activity and Cellular Functions Mediated by Molecularly Evolved Nucleic Acids. Angewandte Chemie International Edition 58, 1621 1625 (2019).

37. Hulme, E. C., Trevethick, M. A. Ligand binding assays at equilibrium: validation and interpretation. British Journal of Pharmacology 161, 1219-1237 (2010).

38. Tian, L., Heyduk, T. Bivalent Ligands with Long Nanometer-Scale Flexible Linkers. Biochemistry 48, 264-275 (2009).

39. Lescoute, A., Westhof, E. Topology of three-way junctions in folded RNAs. RNA 12, 8393 (2006).

40. Mattia, E., Otto, S. Supramolecular systems chemistry. Nature Nanotechnology 10, 111 (2015).

41. Korevaar, P. A., et al. Pathway complexity in supramolecular polymerization. Nature 481, 492 (2012).

42. Wang, J., Liu, K., Xing, R., Yan, X. Peptide self-assembly: thermodynamics and kinetics. Chemical Society Reviews 45, 5589-5604 (2016).

43. Li, G., Zhang, X. C. GTP Hydrolysis Mechanism of Ras-like GTPases. Journal of Molecular Biology 340, 921-932 (2004).

615 44. Lu, K. P., Finn, G., Lee, T. H., Nicholson, L. K. Prolyl cis-trans isomerization as a molecular timer. Nature Chemical Biology 3, 619-629 (2007).

45. Hou, W.-S., Van Parijs, L. A Bcl-2-dependent molecular timer regulates the lifespan and immunogenicity of dendritic cells. Nature Immunology 5, 583-589 (2004).

46. Torabi, S.-F., et al. In vitro selection of a sodium-specific DNAzyme and its application in intracellular sensing. Proceedings of the National Academy of Sciences 112, 59035908 (2015).

47. Reed, M. C., Lieb, A., Nijhout, H. F. The biological significance of substrate inhibition: A mechanism with diverse functions. BioEssays 32, 422-429 (2010).

48. Yoshino, M., Murakami, K. Analysis of the substrate inhibition of complete and partial types. SpringerPlus 4, 292 (2015).

49. Ricci, F., Vallée-Bélisle, A., Simon, A. J., Porchetta, A., Plaxco, K. W. Using Nature's "Tricks" To Rationally Tune the Binding Properties of Biomolecular Receptors. Accounts of Chemical Research 49, 1884-1892 (2016).

50. Andréasson, J., Pischel, U. Molecules with a sense of logic: a progress report. Chemical Society Reviews 44, 1053-1069 (2015).

51. Orbach, R., Remacle, F., Levine, R. D., Willner, I. Logic reversibility and thermodynamic irreversibility demonstrated by DNAzyme-based Toffoli and Fredkin logic gates. Proceedings of the National Academy of Sciences 109, 21228 (2012).

52. Banerjee, A., Bhatia, D., Saminathan, A., Chakraborty, S., Kar, S., Krishnan, Y. Controlled Release of Encapsulated Cargo from a DNA Icosahedron using a Chemical Trigger. Angewandte Chemie International Edition 52, 6854-6857 (2013). 
53. Mariottini, D., Idili, A., Vallée-Bélisle, A., Plaxco, K. W., Ricci, F. A DNA Nanodevice That Loads and Releases a Cargo with Hemoglobin-Like Allosteric Control and Cooperativity. Nano Letters 17, 3225-3230 (2017).

54. Kang, D., Vallée-Bélisle, A., Porchetta, A., Plaxco, K. W., Ricci, F. Re-engineering Electrochemical Biosensors To Narrow or Extend Their Useful Dynamic Range. Angewandte Chemie International Edition 51, 6717-6721 (2012).

55. Porchetta, A., Vallée-Bélisle, A., Plaxco, K. W., Ricci, F. Allosterically Tunable, DNABased Switches Triggered by Heavy Metals. Journal of the American Chemical Society 135, 13238-13241 (2013).

56. Harroun, S. G., et al. Programmable DNA switches and their applications. Nanoscale 10, 4607-4641 (2018).

57. Gao, X. J., Chong, L. S., Kim, M. S., Elowitz, M. B. Programmable protein circuits in living cells. Science 361, 1252 (2018).

650 58. Best, J. A., Nijhout, H. F., Reed, M. C. Homeostatic mechanisms in dopamine synthesis and release: a mathematical model. Theoretical Biology and Medical Modelling 6, 21 (2009).

59. Reed, M., Best, J., Golubitsky, M., Stewart, I., Nijhout, H. F. Analysis of Homeostatic Mechanisms in Biochemical Networks. Bulletin of Mathematical Biology 79, 2534-2557 (2017).

60. Patra, J. K., et al. Nano based drug delivery systems: recent developments and future prospects. Journal of Nanobiotechnology 16, 71 (2018).

61. Lu, H., Wang, J., Wang, T., Zhong, J., Bao, Y., Hao, H. Recent progress on nanostructures for drug delivery applications. Journal of Nanomaterials 2016, (2016). and the origin of new genes in Drosophila species. Nature Genetics 36, 523-527 (2004).

63. Pasek, S., Risler, J.-L., Brézellec, P. Gene fusion/fission is a major contributor to evolution of multi-domain bacterial proteins. Bioinformatics 22, 1418-1423 (2006).

64. Hagner, K., Setayeshgar, S., Lynch, M. Stochastic protein multimerization, activity, and

65. Hashimoto, K., Nishi, H., Bryant, S., Panchenko, A. R. Caught in self-interaction: evolutionary and functional mechanisms of protein homooligomerization. Physical Biology 8, 035007 (2011).

66. Seeman, N. C., Sleiman, H. F. DNA nanotechnology. Nature Reviews Materials 3, 17068 (2017).

67. You, Y., Tataurov, A. V., Owczarzy, R. Measuring thermodynamic details of DNA hybridization using fluorescence. Biopolymers 95, 472-486 (2011).

68. Galau, G. A., Britten, R. J., Davidson, E. H. Studies on nucleic acid reassociation kinetics: rate of hybridization of excess RNA with DNA, compared to the rate of DNA renaturation. Proceedings of the National Academy of Sciences 74, 1020 (1977).

\section{Acknowledgments}

This research was conducted through a Natural Sciences and Engineering Research Council of Canada (NSERC) Discovery Grants (RGPIN-2020-06975) (A.V.-B). A.V.-B. is Canada 
680 Research Chair in Bioengineering and Bionanotechnology, Tier II. D. L. acknowledges a Canada graduate scholarships-master (CGS M) from NSERC and a $3^{\text {rd }}$ cycle scholarship from the Fonds de recherche du Québec - Nature et technologies (FRQNT). The authors would like to thank L. Pedro, K. Nemčeková and S.G. Harroun, for helpful discussions and comments on the paper.

\section{Author contributions}

D.L. and A.V.-B. designed the experiments and D.L. performed all the experiments. D.L and A.V.-B. designed the figures and wrote the paper. 


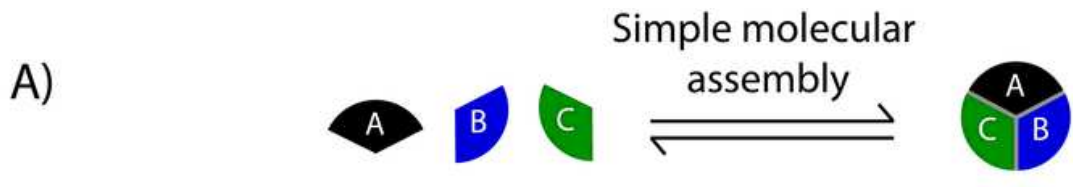

Engineering regulated molecular assembly

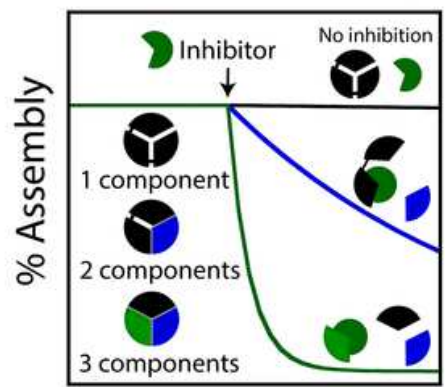

Time

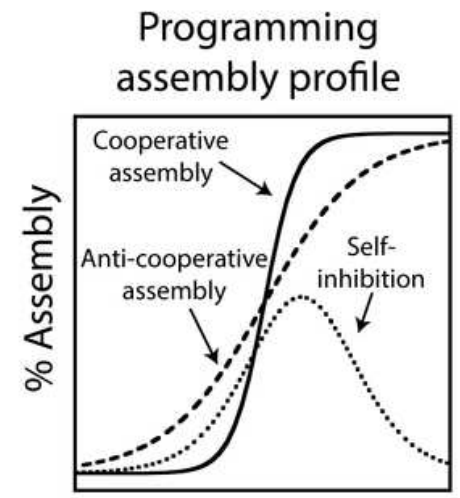

[ब]

C)
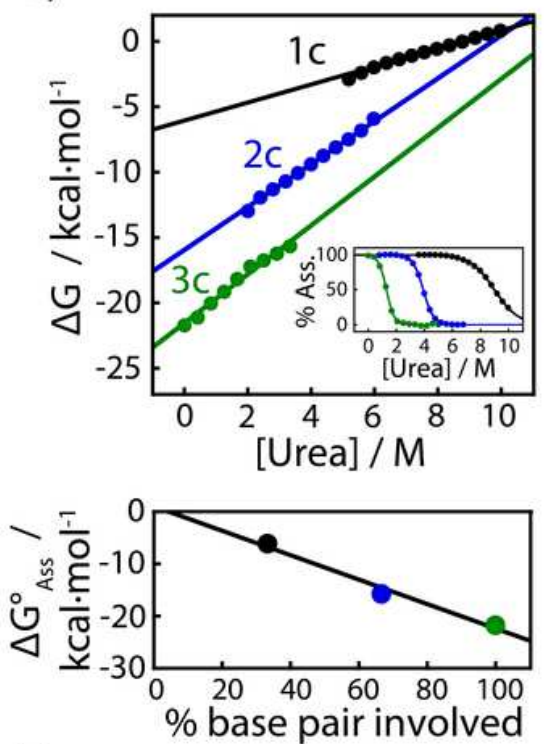

E)

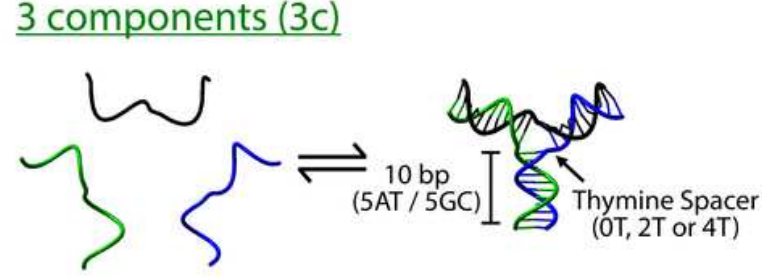

Engineering molecular timer

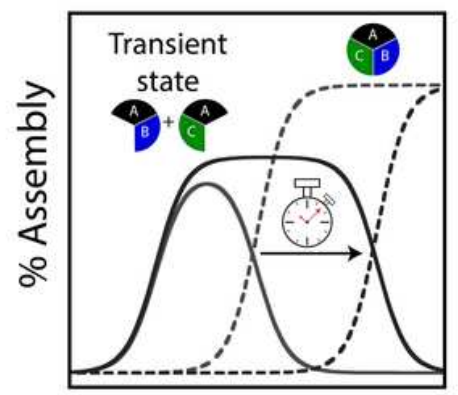

Time

D)
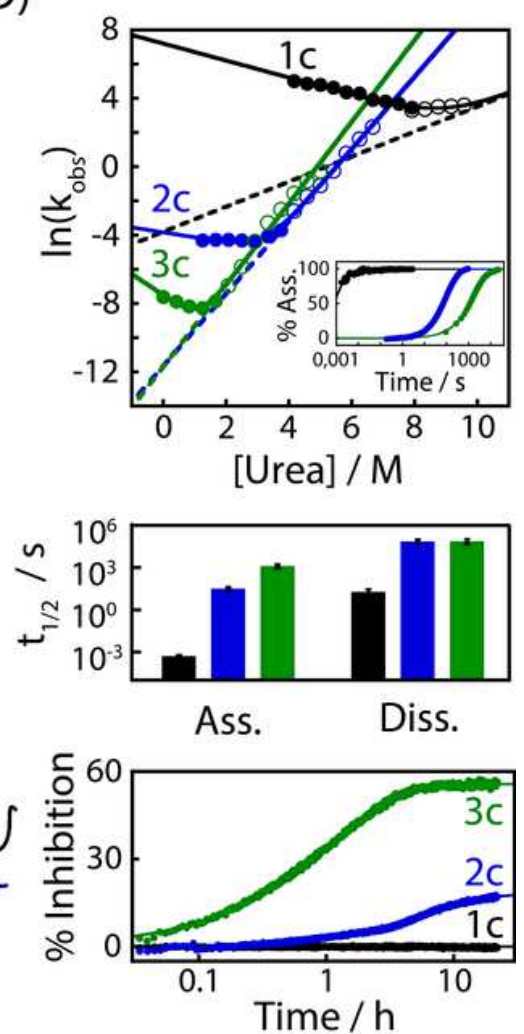

Figure 1

Designing molecular assemblies using one (black, 1c), two (blue, 2c) or three (green, 3c) molecular components. A) Molecular assemblies with multiple components enable the creation of various programmable assembly profiles (e.g., cooperative, anti-cooperative and self-inhibited assembly) with temporally controlled activation (e.g., molecular timer). B) A simple DNA-based self-assembled "3-way junction" nanosystem containing three 10 base pairs (5AT/5GC) arms. All assemblies were monitored 
using fluorescently labeled DNA strands, and the data were normalized accordingly (see Methods). C) Top: Urea (and temperature, Fig. S5) denaturation curves reveal that the free energy of assembly $\left(\Delta \mathrm{G}^{\circ}\right.$ Ass) increases with the number of components: $-5.9 \pm 0.6$ (1c), $-15.8 \pm 0.2$ (2c) and $-21.8 \pm 0.9$ $\mathrm{kcal} / \mathrm{mol}$ (3c) (see inset and Fig. S2 for raw data). Bottom: The $\Delta \mathrm{G}^{\circ}$ Ass and m-values (see Fig. S4) are proportional to the number of base pairs involved in the dissociation process (hairpins remain folded in the dissociated state). D) Top: Kinetics of assembly (closed symbols) and disassembly (open symbols) of all nanosystems under various urea concentrations (chevron plot) reveal a two-state mechanism (see Table S1). Bottom: The 1c system assembles and disassembles faster than the $2 \mathrm{c}$ and $3 \mathrm{c}$ systems (see also Fig. S6-S8 for raw data). E) Increasing the number of components increases the ability to inhibit the assembly process by using a complementary "inhibitor" strand.

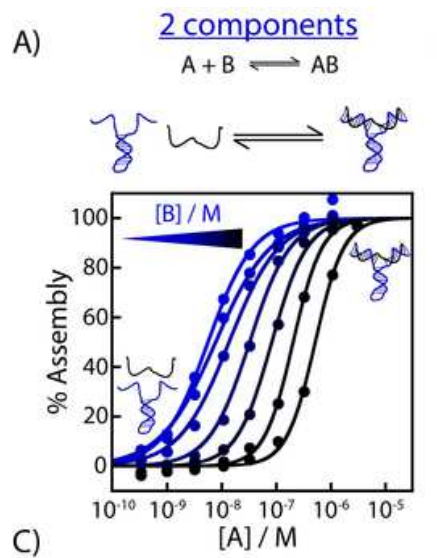

B) $\quad \underline{3 \text { components }}$
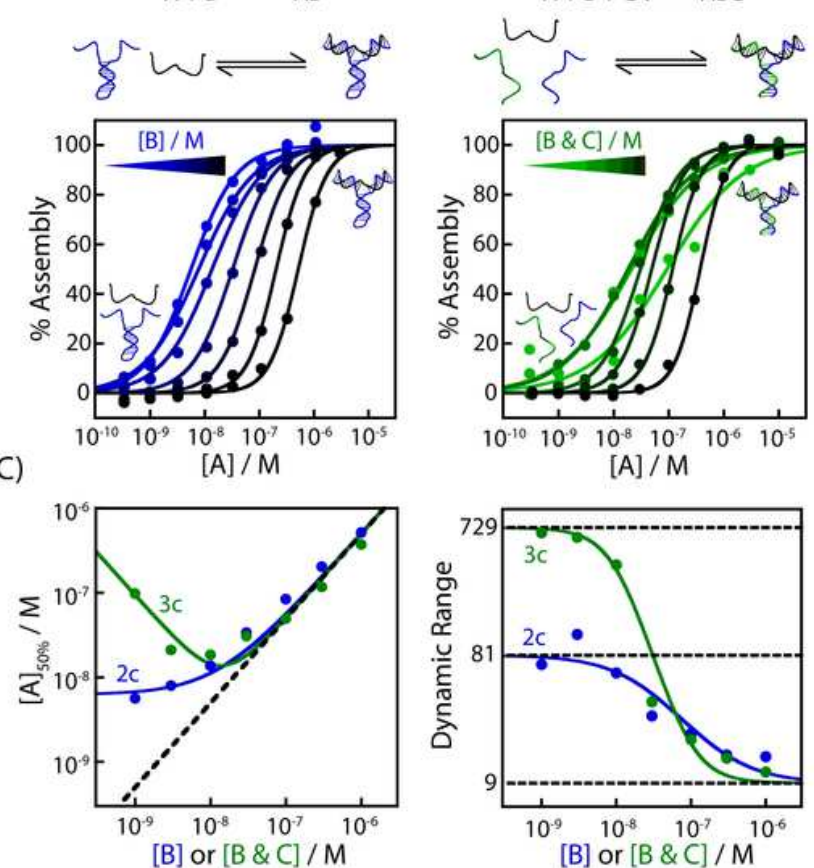

D) Programming the assembly profile of a 3 components nanosystem
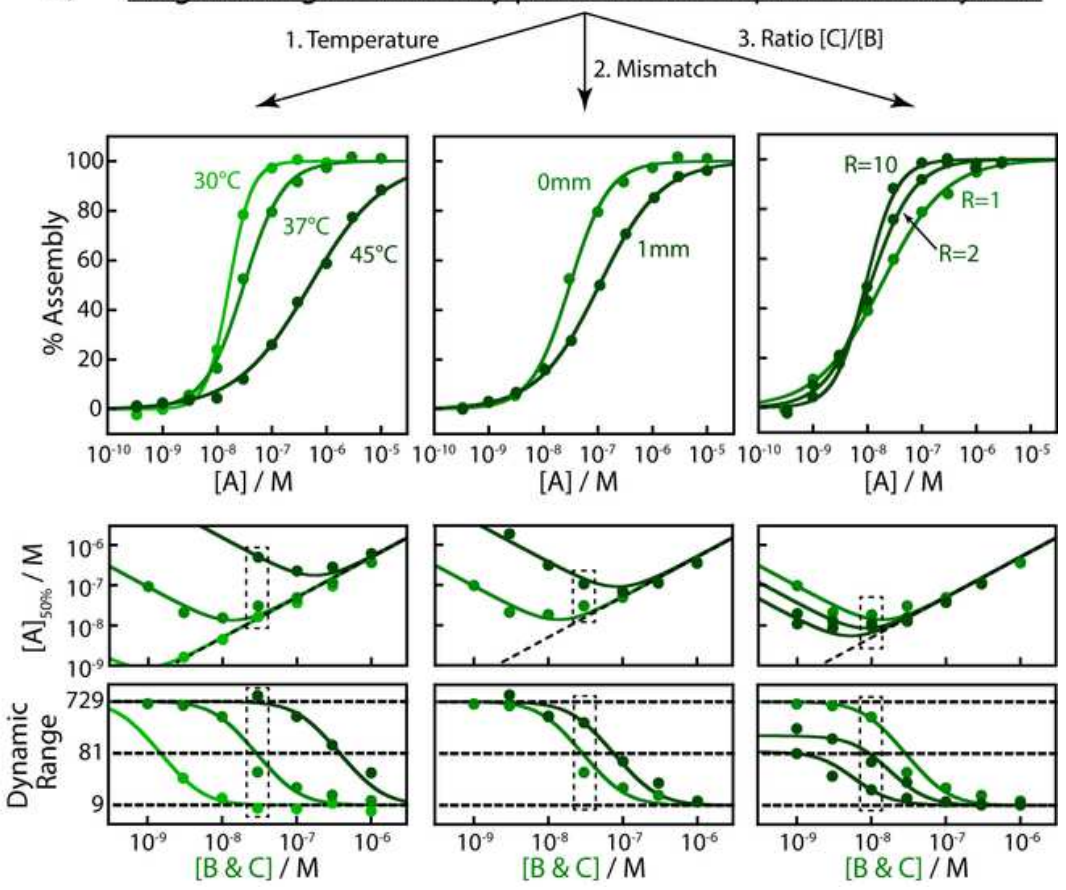

\section{Figure 2}

The assembly of nanosystems becomes more programmable (regulable) as the number of components increases. A) Programming the assembly ([A]50\% and dynamic range) of $2 \mathrm{c}$ and $\mathrm{B}$ ) $3 \mathrm{c}$ systems by increasing $[A]$. Assemblies are preformed at different fixed concentrations of strands $B(2 C)$ or strands $B$ and $C(3 \mathrm{c})$. C) When the concentration of strand $B$ is increased, the $[A] 50 \%$ of the $2 c$ system increases from $5.6 \mathrm{nM}$ to $516 \mathrm{nM}$ (left), while its dynamic range decreases from 81- to 9-fold (right). When the concentrations of both strands $B$ and $C$ are increased, the $[A] 50 \%$ of the $3 c$ system displays a more complex relationship (changing from $92 \mathrm{nM}$ to $18 \mathrm{nM}$ to $372 \mathrm{nM}$-left), while its dynamic range decreases from 729- to 9 -fold (right). D) The assembly profile of 3 components systems can be readily programmed by: 1) changing the temperature (left and Fig. S11); 2) introducing mismatch (middle and Fig. S12) or; 3 ) increasing the concentration ratio between the components B and C (right and Fig. S13). All doseresponse curves are fitted using the Hill equation, while the $[A] 50 \%$ and dynamic range are fitted using equations derived from the $2 \mathrm{c}$ and $3 \mathrm{c}$ models (see supplementary information). The experimental 
conditions were optimized to capture the whole assembly process over the experimentally allowed five orders of magnitude of [A].
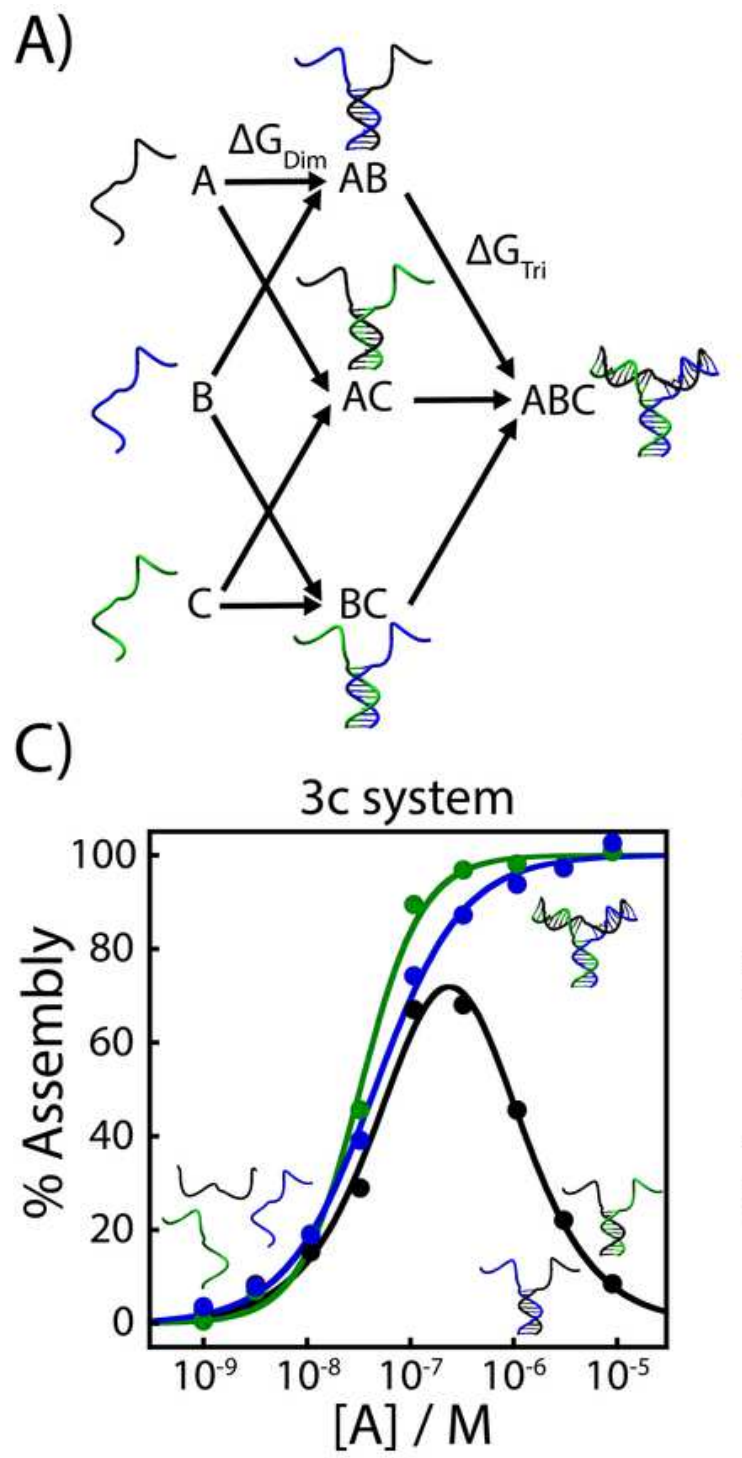

B)

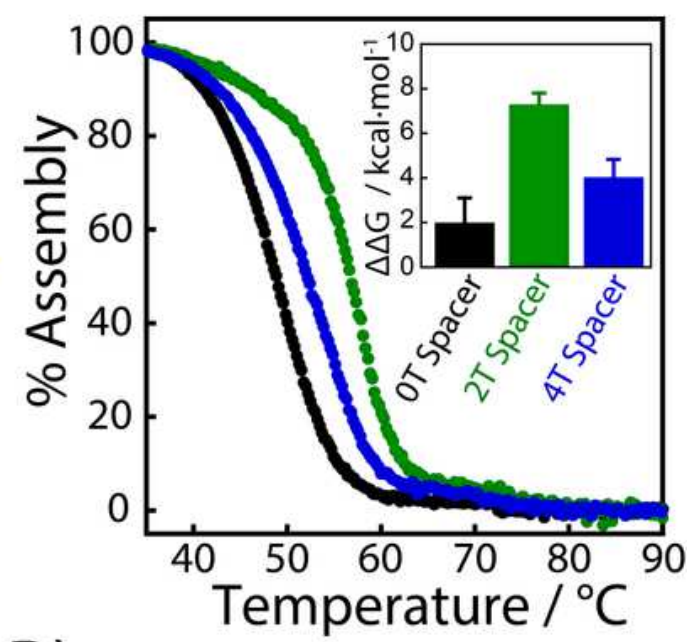

D)

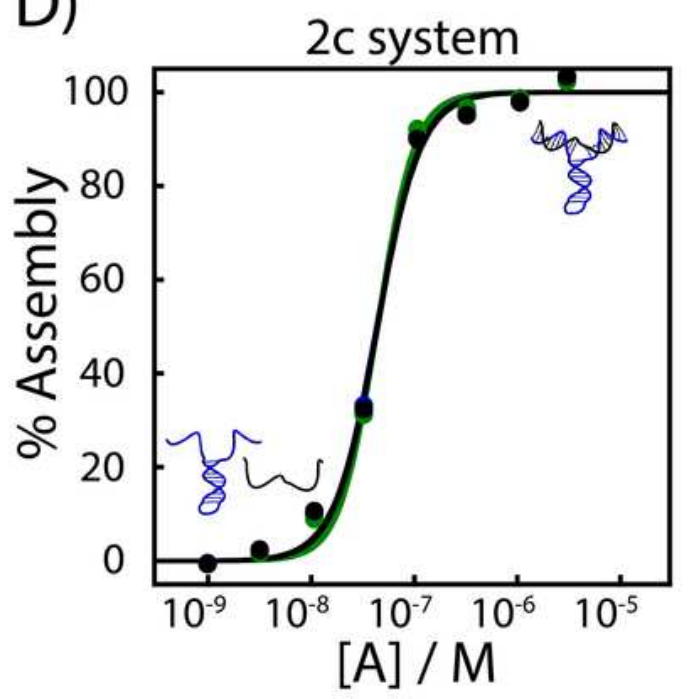

E)

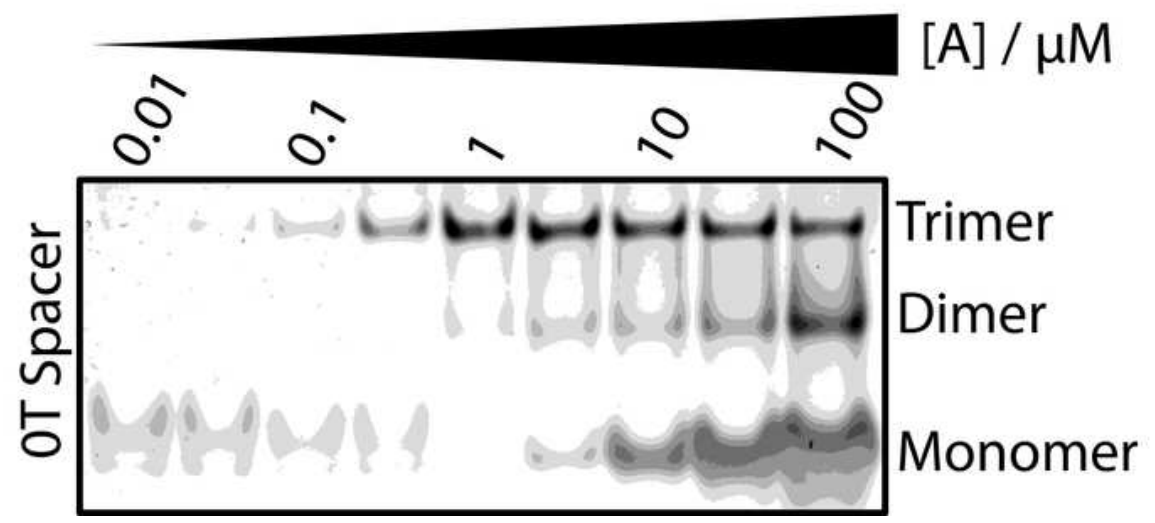

Figure 3

Programming trimer assembly by increasing the difference in energy between the dimer and trimer ( $\triangle \Delta$ GTri-Dim). A) Thermodynamic scheme of the assembly of the 3c systems. B) Changing the thymine spacer length (0T black, $2 \mathrm{~T}$ green and $4 \mathrm{~T}$ blue, see Fig. $1 \mathrm{~A}$ ) creates trimers with different $\Delta \Delta \mathrm{GTri-Dim}$ 
values (see inset). C) Increasing $\Delta \Delta$ GTri-Dim narrows the dynamic range of assembly (as seen with the more cooperative 2T system), while low $\Delta \Delta \mathrm{GTri-Dim}(0 \mathrm{~T})$ creates a self-inhibited trimeric system, which disassembles into two dimers (AB and AC) at higher [A]. D) Similar modifications on the $2 c$ system provide no difference in assembly. E) Polyacrylamide gel electrophoresis of the OT spacer trimeric assembly supports that the decrease in trimer occurs through sequestration into dimers (also see Fig. S15).

A)

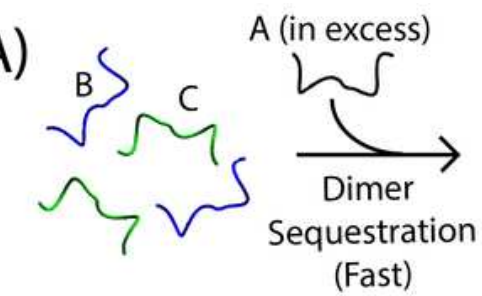

B)

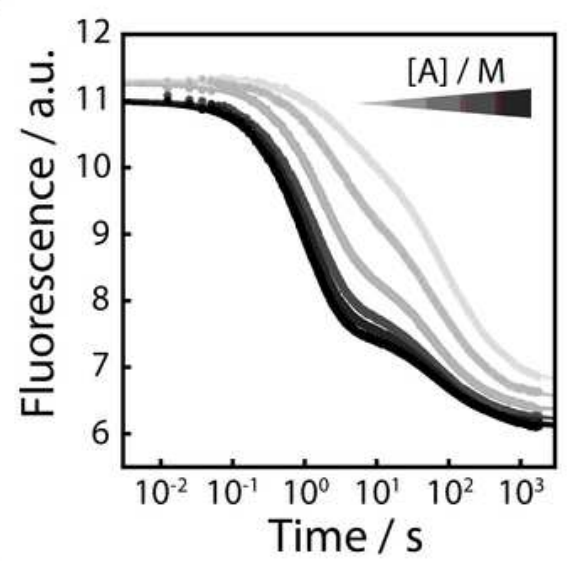

D)

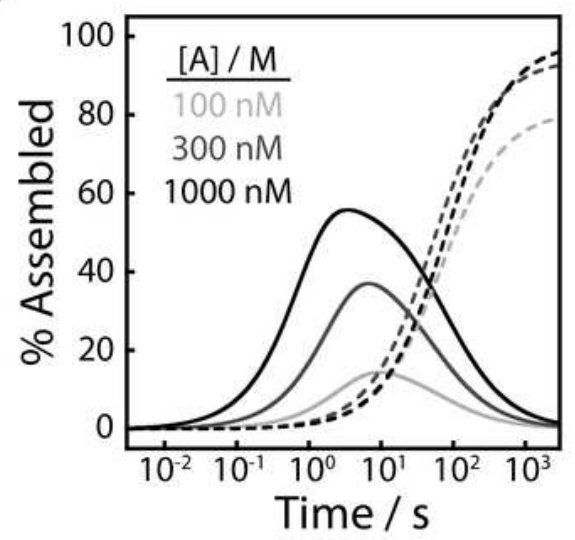

E)
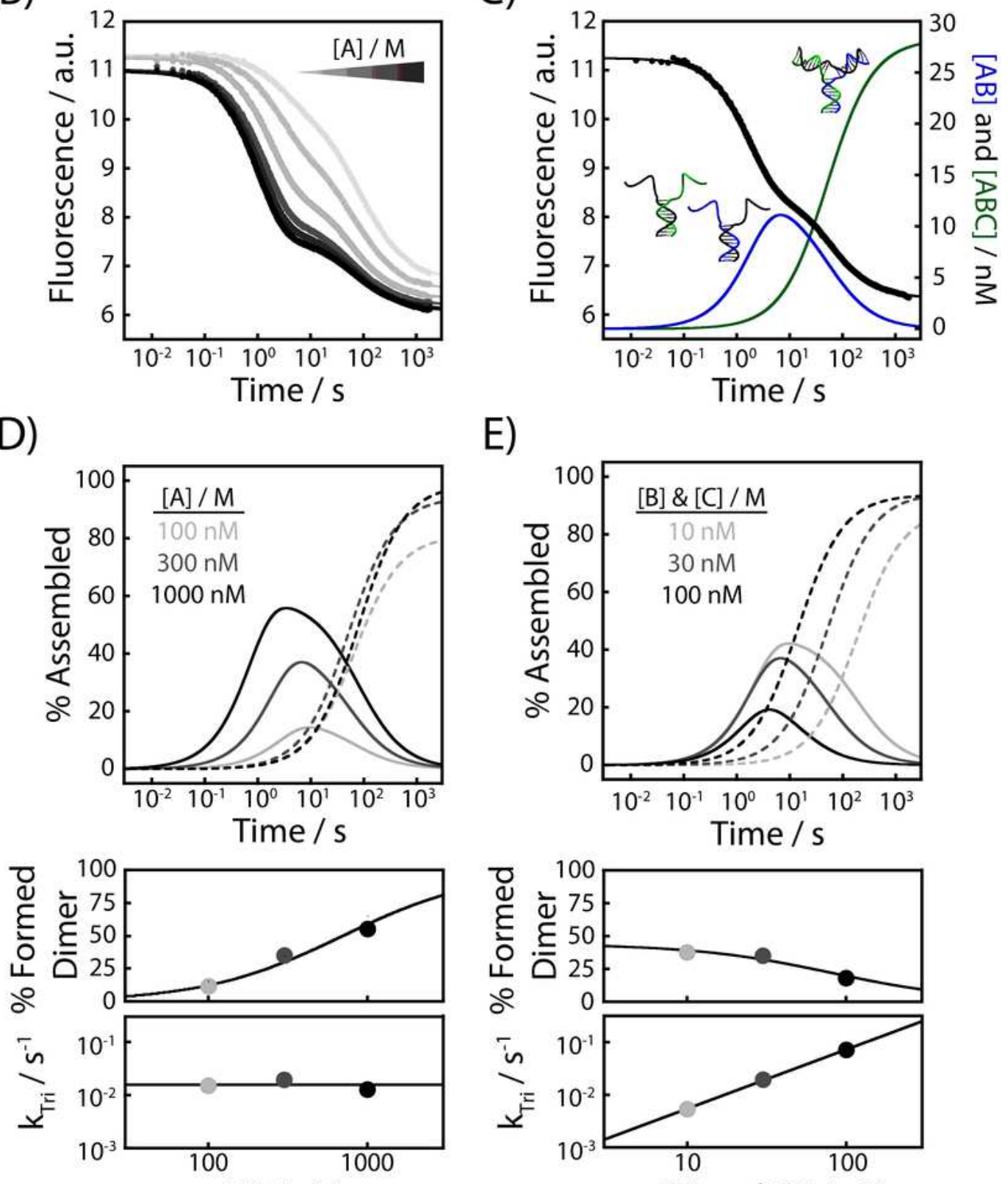

$[\mathrm{A}] / \mathrm{nM}$
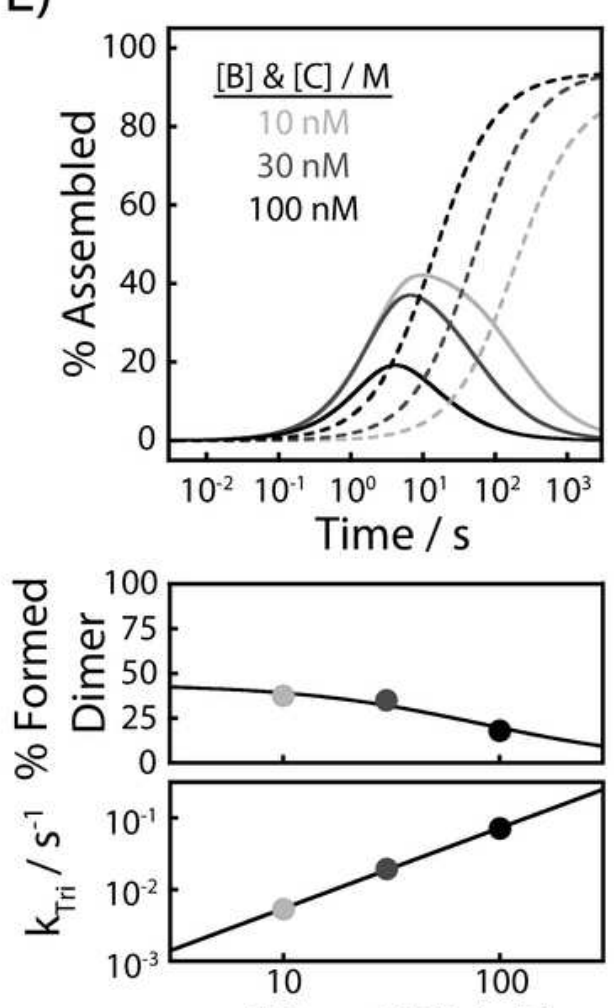

$[\mathrm{B}]$ and $[\mathrm{C}] / \mathrm{nM}$

Figure 4 
Programming time-dependent assembly. A-B) At low concentrations of monomers B and C (mainly unbound), the addition of an excess of monomer $A$ produces slower biphasic kinetics due to the sequestration of strands $B$ and $C$ into $A B$ and $A C$ dimers. Trimer formation then proceeds through a slow strand-exchange mechanism limited by the association of the formerly sequestered components $B$ and $C$ (see Fig. S17 and S20). C) Kinetic trace of the dimeric (blue) and trimeric (green) assemblies derived from the raw kinetic data (black). D) By tuning the concentration of $A$, one can program the rate of formation (activation) and the percentage of formed dimer without affecting the rate of trimeric assembly. E) By increasing the concentrations of $[\mathrm{B}]$ and $[\mathrm{C}]$ and therefore their level of preorganization, one can increase the rate of assembly of the trimer and decrease the percentage of transiently formed dimers. See Fig. S22 for raw data of panels $\mathrm{D}$ and $\mathrm{E}$.

\section{"Two-Component" DNAzyme System}

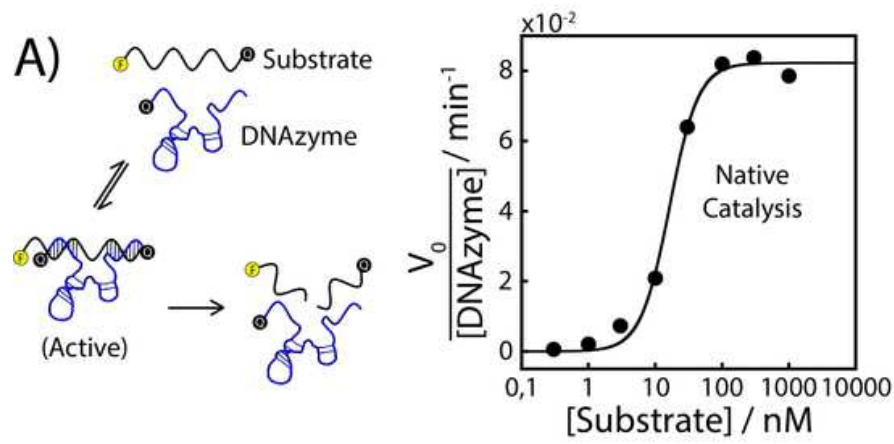

\section{"Three-Component" DNAzyme System (Trimer Active)}

B)
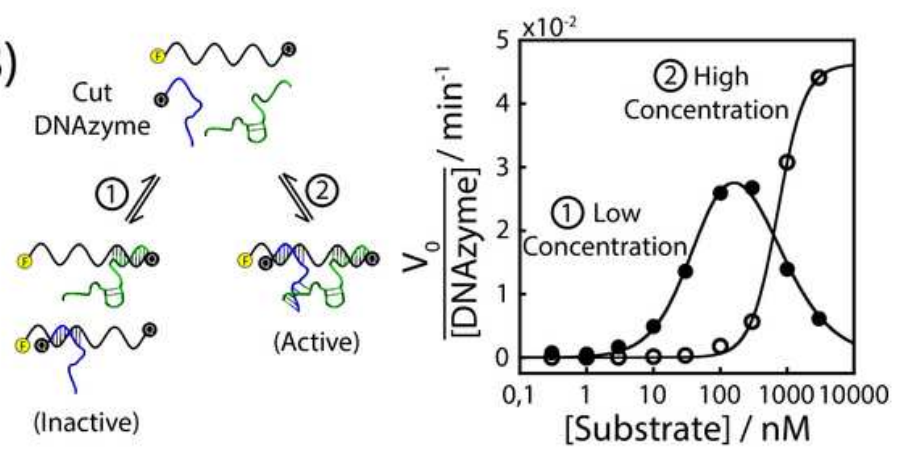

\section{"Three-Component" DNAzyme System (Dimer Active)}

C)

Without Controller

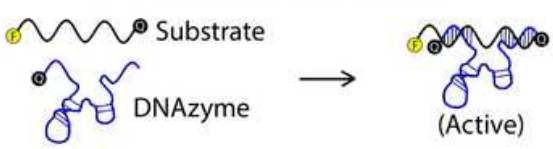

With Controller
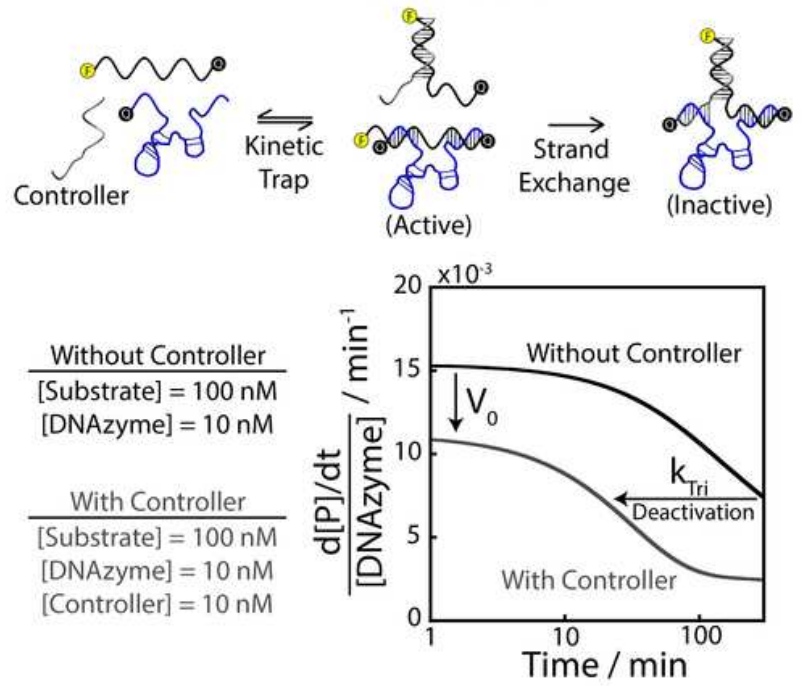

D)
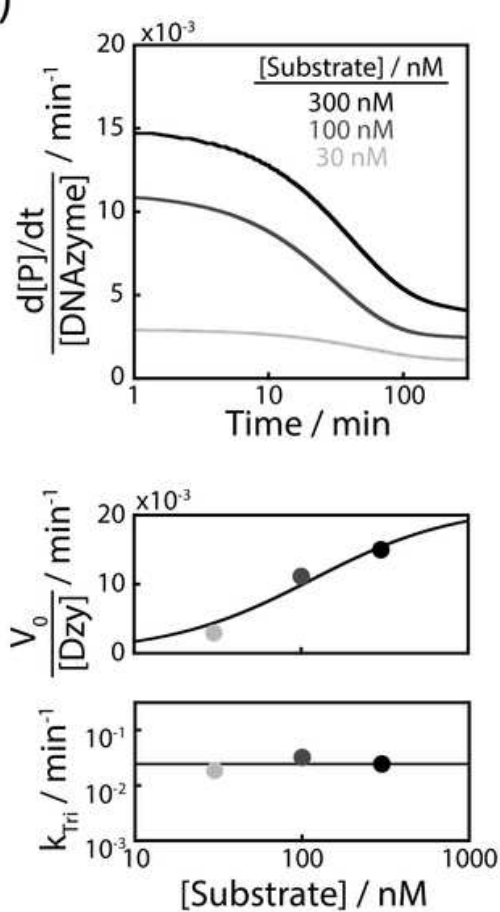

E)
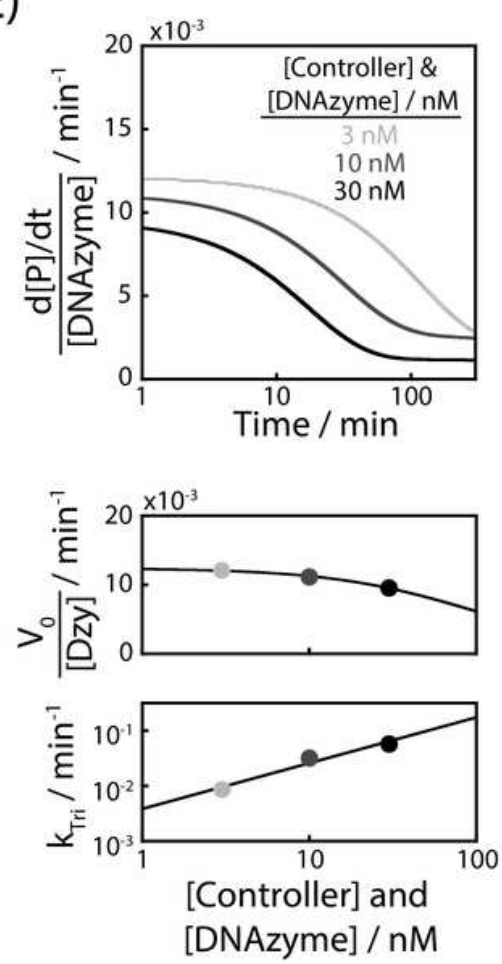

Figure 5 
Figure 5 | Programming complex regulation mechanisms in a catalytic nanosystem through simple molecular assembly. A) The NaA43 cleaving DNAzyme46 displays an [A]50\% of $16 \pm 1 \mathrm{nM}$ at $30 \mathrm{nM}$ DNAzyme and a dynamic range of $9 \pm 1$ consistent with a saturation regime. B) When creating a trimeric assembly by cutting the DNAzyme, one can create a kinetic trap at a low concentration of DNAzyme (30 $\mathrm{nM}$ ) that leads to high dimer sequestration and lower activity. However, if the DNAzyme dimer is already preorganized $(1 \mu \mathrm{M})$, the system once again displays a saturation regime profile ([A]50\% $=729 \pm 29 \mathrm{nM}$ and DR $=7.7 \pm 0.3$ ). For raw data, see Fig. S26. C) We can also create a trimeric assembly by introducing a controller strand that can interact with both the native DNAzyme and the substrate. This controller strand can modulate the level of activity and the deactivation time through the formation of an inactive trimer (bottom). D) In the presence of $10 \mathrm{nM}$ controller and DNAzyme, increasing the concentration of substrate increases the formation of the active dimer and the catalytic rate (top and middle panel) without significantly affecting the trimeric deactivation rate kTri (bottom panel). E) At $100 \mathrm{nM}$ substrate, increasing the concentration of controller and DNAzyme increases the rate of trimer formation and thus the rate of deactivation of the DNAzyme (bottom panel). Of note, the rates shown in panels C-E represent the derivatives of the fitted raw data (see Fig. S29).

\section{Supplementary Files}

This is a list of supplementary files associated with this preprint. Click to download.

- SupplementaryInformationAVB.docx 